\title{
Atomic electric dipole moments: The Schiff theorem and its corrections
}

\author{
C.-P. Liu, ${ }^{1,2}$ M. J. Ramsey-Musolf, ${ }^{3,4}$ W. C. Haxton,${ }^{5}$ R. G. E. Timmermans, ${ }^{2}$ and A. E. L. Dieperink ${ }^{2}$ \\ ${ }^{1}$ T-16, Theoretical Division, Los Alamos National Laboratory, Los Alamos, New Mexico 87545, USA \\ ${ }^{2}$ Theory Group, Kernfysisch Versneller Instituut, University of Groningen, Zernikelaan 25, 9747 AA Groningen, The Netherlands \\ ${ }^{3}$ Kellogg Radiation Laboratory, California Institute of Technology, Pasadena, California 91125, USA \\ ${ }^{4}$ Department of Physics, University of Wisconsin-Madison, Madison, Wisconsin 53706, USA \\ ${ }^{5}$ Institute for Nuclear Theory and Department of Physics, University of Washington, Box 351550, Seattle, Washington 98195-1550, USA
}

(Received 14 May 2007; published 24 September 2007)

\begin{abstract}
Searches for the permanent electric dipole moments (EDMs) of diamagnetic atoms provide powerful probes of CP-violating hadronic and semileptonic interactions. The theoretical interpretation of such experiments, however, requires careful implementation of a well-known theorem by Schiff that implies a vanishing net EDM for an atom built entirely from pointlike, nonrelativistic constituents that interact only electrostatically. Any experimental observation of a nonzero atomic EDM would result from corrections to the pointlike, nonrelativistic, electrostatic assumption. We reformulate Schiff's theorem at the operator level and delineate the electronic and nuclear operators whose atomic matrix elements generate corrections to "Schiff screening." We obtain a form for the operator responsible for the leading correction associated with finite nuclear size-the so-called Schiff moment operator-and observe that it differs from the corresponding operator used in previous Schiff moment computations. We show that the more general Schiff moment operator reduces to the previously employed operator only under certain approximations that are not generally justified. We also identify other corrections to Schiff screening that may not be included properly in previous theoretical treatments. We discuss practical considerations for obtaining a complete computation of corrections to Schiff screening in atomic EDM calculations.
\end{abstract}

DOI: 10.1103/PhysRevC.76.035503

PACS number(s): 24.80.+y, 21.10.Ky, 11.30.Er, 27.80.+w

\section{INTRODUCTION}

The search for $\mathrm{CP}$ violation (CPV) in and beyond the Standard Model (SM) is important to particle, nuclear, and atomic physics and their intersections with cosmology. The $\mathrm{CPV}$ in the electroweak sector of the SM, parametrized by the complex phase in the Cabibbo-Kobayashi-Maskawa (CKM) matrix, adequately accounts for experimental observations of $\mathrm{CPV}$ effects in neutral kaon and $B$ meson systems. The results of searches for the permanent electric dipole moments (EDMs) of the neutron $\left(d_{n}\right)$ and diamagnetic atoms $\left(d_{A}\right)$ imply that CPV in the strong sector of the SM, parametrized by the so-called $\theta$-term, is vanishingly small: $|\bar{\theta}| \lesssim 10^{-10}[1,2]$. The so-called strong $\mathrm{CP}$ problem associated with the unnaturally small value of $\bar{\theta}$ may find resolution in spontaneously broken Peccei-Quinn symmetry if the corresponding Goldstone boson - the axionis found. If so, strong CPV may hold the key to one of the outstanding puzzles in cosmology, as the axion is a viable candidate for particle dark matter.

Nevertheless, another cosmological problem - the origin of the nonvanishing baryon asymmetry of the universe (BAU) would remain unsolved. As noted by Sakharov nearly four decades ago [3], if the initial conditions of the universe were matter-antimatter symmetric, the particle physics of the evolving universe would have to contain CPV interactions (along with baryon number violation and a departure from thermal equilibrium) to produce a BAU. It has been subsequently noted that the CPV contained in the SM is not sufficiently effective to produce the observed $\mathrm{BAU}$, so an explanation requires new sources of CPV. If such interactions involve particles having masses $\lesssim \mathrm{a}$ few $\mathrm{TeV}$, then current searches for atomic, hadronic, and leptonic EDMs could soon yield nonzero results. Existing limits already provide stringent constraints on new electroweak-scale CPV, and the next generation of experiments is poised to push the sensitivity to new CPV sources by several orders of magnitude. If new electroweak-scale CPV in models such as supersymmetry is, indeed, responsible for the BAU, then one would expect nonzero results in this new round of EDM searches (see, e.g., Ref. [4] and references therein). For a recent review of EDMs and CPV, see Ref. [5].

If a nonzero EDM is observed, then it will take a set of complementary EDM experiments- together with the results of future collider searches and precision electroweak studiesto identify the nature of CPV. In this respect, searches for EDMs of diamagnetic atoms play a key role. Experimentally, new efforts to probe $d_{A}$ with Ra [6,7], Xe [8], and Rn [9] are presently underway. The sensitivity of these atomic EDMs to either $|\bar{\theta}|$ or CPV beyond that of the SM could exceed that obtained for the ${ }^{199} \mathrm{Hg}$ atom [2], which currently sets the standard for $d_{A}$, by several orders of magnitude. The ${ }^{199} \mathrm{Hg}$ standard is also expected to be improved by an order of magnitude when current experiments are completed [10]. Theoretically, the interpretation of $d_{A}$ is challenging because of the interplay of atomic, nuclear, and hadronic physics. Specifically, it has long been recognized that an EDM residing on the nucleus of a neutral diamagnetic atom would not be detectable, apart from small corrections: Schiff [11] showed that any neutral system of electrically charged, nonrelativistic, pointlike constituents interacting only electrostatically will have no net EDM. ${ }^{1}$ Schiff's result can be understood at the classical level. An atomic EDM is probed by placing the atom

\footnotetext{
${ }^{1}$ In fact, part of this shielding effect was pointed out by Purcell and Ramsey [12], but it is Schiff who first clearly demonstrates this effect in quantum mechanics.
} 
in a combination of external magnetic and electric fields. If the atom has a net EDM, then its interaction with the external electric field $\boldsymbol{E}_{\text {ext }}$ would lead to a shift in the Larmor precession frequency that depends linearly on $\boldsymbol{E}_{\text {ext }}$. However, a neutral system in the presence of an external electric field cannot accelerate. Thus, the atomic constituents-assumed here to be pointlike-must rearrange so as to screen out the effect of $\boldsymbol{E}_{\text {ext }}$ at the location of each of the charged constituents. Consequently, if any of the charged constituents (electrons or nucleus) possesses a nonzero EDM, changes in the interaction energy owing to $\boldsymbol{E}_{\text {ext }}$ will be exactly canceled by those from the internal fields induced by atomic polarization. To the extent that the interaction of the atom with $\boldsymbol{E}_{\text {ext }}$ arises solely from the EDMs of its pointlike, nonrelativistic constituents, there will be no shift in the Larmor frequency linear in $\boldsymbol{E}_{\text {ext }}$ and, hence, no effective atomic EDM. Corrections to this exact screening will arise if any of the constituents having an EDM (a) moves relativistically, leading to a breakdown of the classical picture, (b) has a finite size, leading to a breakdown of the pointlike assumption, or (c) has nonelectrostatic interactions that become modified in the presence of the atomic rearrangement needed to achieve electrostatic equilibrium. In addition, CPV interactions between constituents can lead to an atomic EDM that evades the Schiff screening.

Because of such corrections and the exquisite sensitivity of the measurements, atomic EDM experiments place important constraints on the electron EDM and on CPV interactions in the nucleus. The present limits on the electron EDM $d_{e}$, for example, are derived from heavy paramagnetic atoms, where the Schiff theorem is evaded by the relativistic motion of the atomic electrons. In the case of diamagnetic atoms, nonzero effects from hadronic CPV arise because of corrections (b) and (c), specifically the finite size of the nucleus that leads to imperfect shielding and magnetic and other, higher-order, multipole interactions between the nucleus and atomic electrons. The finite-size correction becomes much more important in heavier atoms: To the extent that the atomic electrons penetrate the atomic nucleus, a residual CPV interaction arises. This penetration produces an atomic EDM proportional to the so-called Schiff moment $\langle\hat{\boldsymbol{S}}\rangle$ of the nucleus, often expressed as an $r^{3}$-weighted integral over the difference in the nuclear charge and EDM distributions. Additional contributions to the atomic EDM are generated by hyperfine (nonelectrostatic) interactions between atomic electrons and $\mathrm{CP}$-conserving higher-order nuclear moments, such as $M_{1}$ (magnetic dipole) and $C_{2}$ (charge quadrupole), as well as higher-order CPV nuclear moments that couple to spatially varying fields, such as $M_{2}$ (magnetic quadrupole).

These general features of Schiff screening and its corrections were identified in Schiff's original paper and subsequently discussed in other work by Sandars [13], Feinberg [14], and Sushkov, Flambaum, and Khriplovich [15] (for later discussions, see, e.g., Refs. [16-20]). The formulation of the corrections to Schiff screening in Refs. $[14,15]$ concentrated on the Schiff moment effect, and approximate expressions for $\hat{S}$ were derived. The latter have been used in subsequent theoretical treatments of the EDMs of ${ }^{199} \mathrm{Hg}$ and other atoms, from which limits on CPV parameters, such as $|\bar{\theta}|$, have been derived (see, e.g., Ref. [21] and references therein).
Although these treatments are correct at a qualitative level, more precise derivations are possible and are likely important given the prospect that nonzero atomic EDMs may soon be seen. In what follows, we provide a systematic derivation of Schiff's theorem and the associated corrections in a spherical multipole formalism. When comparisons are made to earlier work, several refinements are apparent:

(i) Our approach expresses the electron-nucleus interaction in terms of spherical multipole operators, separating contributions associated with electron penetration from those associated with a pointlike nucleus. In so doing, we obtain a form for the atomic operator $\hat{O}_{\text {Schiff }}^{\text {atomic }}$ that describes the leading corrections to Schiff screening associated with electronic penetration into the nucleus. This atomic operator can be applied to any atomic EDM and gives a more general characterization of the leading finite-size corrections than has been used in previous studies.

(ii) We subsequently derive the nuclear Schiff moment operator $\hat{\boldsymbol{S}}$ by considering matrix elements of $\hat{O}_{\text {Schiff }}^{\text {atomic }}$ between electronic $\mathrm{S}$ and $\mathrm{P}$ states as is relevant for many atomic EDMs. Our result for $\hat{\boldsymbol{S}}$, the analog of the Schiff moment operator used in many earlier studies, has a more general form than the one in common use. We discuss the approximations under which our expression for $\hat{S}$ reduces to the form conventionally employed, and we observe that these approximations are not generally justified. We advocate the use of the operator $\hat{S}$ derived in the following that does not require adoption of any approximation. We also note that the operator $\hat{\boldsymbol{S}}$ derived for electronic S-P transitions is not appropriate for other transitions, such as the close-lying D-P transition in a metastable Ra atom where a large enhancement factor is found [22].

(iii) We derive expressions for the operators that characterize interactions between the polarized atomic cloud and CP-conserving nuclear moments, such as the magnetic dipole and charge quadrupole moments. We also include finite-size, electronic penetration effects associated with these multipole moments.

(iv) We obtain additional electronic penetration corrections involving higher CPV multipole moments of the nuclear charge and current densities, such as the charge octupole moment and magnetic quadrupole moment.

(v) We revisit corrections to Schiff screening of electron EDMs and include corrections associated with both the external and nuclear vector potentials that have not been included in earlier studies.

In addition to deriving the operators associated with the foregoing effects, we also discuss practical considerations involved in computing atomic EDMs. For example, a complete computation of effects associated with hadronic CPV would require a simultaneous diagonalization of the electronic and nuclear Hamiltonian, taking into account effects such as the nuclear response to the polarized atomic cloud and the corresponding interaction with the external electric field. Practically speaking, carrying out such a diagonalization in the full space of atomic wave functions is not possible 
even in the limit of purely $\mathrm{CP}$-conserving interactions. In typical atomic calculations, the nuclear charge and current distributions are taken as $c$-number sources to which the atomic electrons respond, and "back reactions" of the nuclear charge and current distributions to the atomic electrons- the so-called nuclear polarization corrections-are neglected. This procedure amounts to treating the atomic states as direct products of the nuclear ground state and different electronic states, with the properties of the nuclear ground state acting as a $c$-number input for diagonalization of the electronic Hamiltonian:

$$
\left.|n\rangle_{\text {atom }}=\mid \text { g.s. }\right\rangle_{\mathcal{N}} \otimes|k\rangle_{e},
$$

where the $\mathcal{N}$ and $e$ subscripts refer to the nuclear ground state and electronic states (labeled by quantum numbers $k$ ), respectively.

One would Naïvely expect the nuclear polarization corrections to atomic properties associated with this factorization to be small, going as powers of the nuclear radius, $R_{\mathcal{N}}$, divided by the effective Bohr radius, $a_{0}$. For electronic $\mathrm{S}$ states, however, the situation is more subtle. Theoretical studies of muonic atoms, for which the effective Bohr radius is $\sim 1 / 200$ of that for ordinary atoms, indicate that corrections to the energy splitting between the $2 \mathrm{~S}_{1 / 2}$ and $2 \mathrm{P}_{3 / 2}$ states depends on the square of the muon wave function at the origin and an integral over the nuclear photoabsorption cross section [23-25]. Numerically, the theoretical polarization correction to $\Delta E\left(2 \mathrm{P}_{3 / 2}-2 \mathrm{~S}_{1 / 2}\right)$ in $\left(\mu^{4} \mathrm{He}\right)^{+}$is about $0.2 \%$ of the measured splitting. In the case of $\left(e^{4} \mathrm{He}\right)^{+}$, the authors of Refs. [23,24] find that the nuclear polarization correction to the S-P splitting is a few $\times 10^{-4}$ times the magnitude of the corresponding finite-size correction, which depends on the square of the electronic wave function at the origin times the mean-square nuclear charge radius.

The Schiff moment interaction itself characterizes finitesize corrections of order $\left(R_{\mathcal{N}} / a_{0}\right)^{2}$ relative to the energy associated with the interaction of the atomic electrons with the EDM of a pointlike nucleus. Based on the studies of Refs. [23-25], one would expect the nuclear polarization corrections to the finite-size effects characterized by the Schiff moment to be negligible. In the present analysis, however, we have not quantified these polarization effects, leaving this task to future work. Instead, we will proceed by formulating the Schiff theorem at the operator level as far as possible without making explicit reference to the atomic states or invoking the factorization approximation, thereby avoiding the issue of nuclear polarization corrections. Only when attempting to compare our Schiff moment operator with the corresponding operator used previously in the literature will we adopt the factorization ansatz of Eq. (1). Even in this case, we find substantial differences with the Schiff moment operator used in earlier analyses.

With the foregoing caveats in mind, we believe that our reformulation of Schiff's theorem represents a useful refinement that will allow for a more complete inclusion of hadronic and nuclear structure contributions to $d_{A}$ and provide for a sharper confrontation between theory and experiment. We organize our reformulation in the remainder of the paper as follows. In Sec. II we give our multipole operator formulation of the theorem, including a delineation of the various operators that characterize corrections to Schiff screening. For completeness, we include here a discussion of both the hadronic CPV effects as well as those associated with electronic EDMs. We include here the corrections associated with nonelectrostatic interactions between atomic electrons and both nuclear and external sources, which are ignored in previous studies. In Sec. III, we obtain the full atomic Schiff moment operator $\hat{O}_{\text {Schiff }}^{\text {atomic }}$, which is independent of the atomic states, and use it, together with the factorization ansatz, to derive an effective nuclear Schiff moment operator $\hat{S}$ that can be compared with the operator used previously in the literature. We also derive the operator that characterizes the leading corrections to Schiff screening associated with magnetic interactions between the electrons and nucleus. In Sec. IV, we summarize our results and discuss the practical implementation of Schiff's theorem including the issues to which we have already alluded. A few technical details are given in the Appendices.

\section{SCHIFF'S THEOREM: A MULTIPOLE OPERATOR FORMULATION}

The interaction of an external field with the atomic EDM is odd under both parity $(\mathrm{P})$ and time-reversal $(\mathrm{T})$ transformations but even under charge conjugation (C). Assuming CPT invariance, as we do throughout this paper, we have that the observation of a nonzero $d_{A}$-induced frequency shift is, thus, equivalent to the observation of $\mathrm{CP}$ violation. Henceforth, we will refer to atomic, electronic, and nuclear moments that violate both $\mathrm{P}$ and $\mathrm{T}$ symmetries as PVTV moments; those that respect these symmetries are PCTC moments. Effects that violate $\mathrm{P}$ but conserve $\mathrm{T}$, such as the nuclear anapole moment, are also of considerable interest, but we do not consider these effects here (for recent reviews, see Refs. [26,27]). Effects that conserve $\mathrm{P}$ but violate $\mathrm{T}$, which in combination with the weak interaction can induce PVTV interactions, are also not explicitly treated here.

\section{A. The atomic Hamiltonian}

To evaluate the consequences of PVTV interaction of atomic properties, we separate the complete atomic Hamiltonian into PCTC $\left(H_{0}\right)$ and PVTV $\left(H_{I}\right)$ terms and treat the latter as a perturbation:

$$
H_{\text {atom }}=H_{0}+H_{I} \text {. }
$$

We also include the interaction of an external field with the atom in $H_{I}{ }^{2}$ Before identifying the specific operators in $H_{0}$ and $H_{I}$, it is useful to delineate the different interactions among atomic electrons, the electrons and nucleus, and external fields and the atomic constituents:

(i) The interaction between atomic electrons is

$$
H_{\mathrm{int}}^{(e e)}=V_{\mathrm{int}}^{(e e)}+\tilde{V}_{\mathrm{int}}^{(\tilde{e} e)},
$$

\footnotetext{
${ }^{2}$ As we are interested in permanent EDMs instead of induced EDMs, it is the first-order Stark effect that one is after. Therefore, the limit of weak external field is adequate.
} 
where we adopt the notation (used throughout this paper) where the superscript (ee) denotes a PCTC electron-electron interaction with PCTC couplings on both electrons, and (ẽe) denotes a PVTV interaction that arises from a PCTC coupling on one electron and a PVTV coupling on the other. Specifically, $V_{\text {int }}^{(e e)}$ denotes the PCTC Coulomb and Breit interactions between electrons,

$$
V_{\mathrm{int}}^{(e e)}=\frac{\alpha}{2} \sum_{i=1}^{Z}\left(\phi_{i}^{(e)}-\boldsymbol{\alpha}_{i} \cdot \boldsymbol{A}_{i}^{(e)}\right),
$$

and $\tilde{V}_{\text {int }}^{(\tilde{e} e)}$ denotes the PVTV electron-electron interaction, which we write as

$$
\tilde{V}_{\text {int }}^{(\tilde{e} e)}=\frac{\alpha}{2} \sum_{i=1}^{Z} d_{e} \beta\left[\boldsymbol{\sigma}_{i} \cdot \boldsymbol{E}_{i}^{(e)}+i \boldsymbol{\alpha}_{i} \cdot \boldsymbol{B}_{i}^{(e)}\right]+\cdots .
$$

The term given explicitly is the interaction of each electron's EDM with the electric and magnetic fields $\left(\boldsymbol{E}_{i}^{(e)}, \boldsymbol{B}_{i}^{(e)}\right)$ created by the other electrons. In addition, the " $+\cdots$ " indicates that possible additional terms, that is, new exchanges generating PVTV interactions, could be added to this. Here, $\alpha$ is the fine structure constant and $\beta_{i}$ and $\alpha_{i}$ are the Dirac matrices acting on the $i$ th electron. The scalar potential $\phi_{i}^{(e)}=\phi^{(e)}\left(\boldsymbol{x}_{i}\right)$ is the potential exerted by the other $Z-1$ electrons at the position $\boldsymbol{x}_{i}$ of the $i$ th electron; the notation is similar for the vector potential $\boldsymbol{A}_{i}^{(e)}$ and for the resulting electric and magnetic fields $\boldsymbol{E}_{i}^{(e)}$ and $\boldsymbol{B}_{i}^{(e)}$. The 1/2 factor in the electron-electron interaction is introduced to ensure that one sums over distinct pairwise interactions. ${ }^{3}$

(ii) The interaction between the electrons and nucleus $(\mathcal{N})$ is

$$
H_{\mathrm{int}}^{(e \mathcal{N})}=-\alpha \sum_{i=1}^{Z}\left[\phi_{i}^{(\mathcal{N})}-\boldsymbol{\alpha}_{i} \cdot \boldsymbol{A}_{i}^{(\mathcal{N})}\right]+\tilde{V}_{\mathrm{int}}^{(\tilde{\mathcal{e}})},
$$

where $\phi_{i}^{(\mathcal{N})}$ and $\boldsymbol{A}_{i}^{(\mathcal{N})}$ are the scalar and vector potentials of the nucleus at the position of the $i$ th electron. These potentials, as discussed in the following, in general contain both PCTC and PVTV contributions. The second term, $\tilde{V}_{\text {int }}^{(\widetilde{e})}$, is the PVTV contribution owing to the interaction of electron EDMs with the PCTC electric and magnetic fields $\left(\boldsymbol{E}_{i}^{(\mathcal{N})}, \boldsymbol{B}_{i}^{(\mathcal{N})}\right)$ associated with the nuclear potentials,

$\tilde{V}_{\mathrm{int}}^{(\tilde{\mathcal{N}})}=-\alpha \sum_{i=1}^{Z} d_{e} \beta\left[\boldsymbol{\sigma}_{i} \cdot \boldsymbol{E}_{i}^{(\mathcal{N})}+i \boldsymbol{\alpha}_{i} \cdot \boldsymbol{B}_{i}^{(\mathcal{N})}\right]+\cdots$.

Again, the " $+\ldots$ " indicates additional PVTV nonelectromagnetic exchanges that might exist between the electrons and the nucleus.

\footnotetext{
${ }^{3}$ Throughout this paper, we factor out electric charge from the scalar and vector potentials.
}

(iii) The interaction of the electrons with the applied

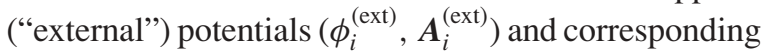
fields $\left(\boldsymbol{E}_{i}^{(\text {ext) }}, \boldsymbol{B}_{i}^{(\text {ext) }}\right)$ is

$$
H_{\mathrm{ext}}^{(e)}=V_{\mathrm{ext}}^{(e)}+\tilde{V}_{\mathrm{ext}}^{(\tilde{e})},
$$

where

$$
\begin{aligned}
& V_{\mathrm{ext}}^{(e)}=-\alpha \sum_{i=1}^{Z}\left(\phi_{i}^{(\mathrm{ext})}-\boldsymbol{\alpha}_{i} \cdot \boldsymbol{A}_{i}^{(\mathrm{ext})}\right) \\
& \tilde{V}_{\mathrm{ext}}^{(\tilde{e})}=-\alpha \sum_{i=1}^{Z} d_{e} \beta\left(\boldsymbol{\sigma}_{i} \cdot \boldsymbol{E}_{i}^{(\mathrm{ext})}+i \boldsymbol{\alpha}_{i} \cdot \boldsymbol{B}_{i}^{(\mathrm{ext})}\right) .
\end{aligned}
$$

(iv) The interaction of the nucleus with the external potentials is

$$
H_{\mathrm{ext}}^{(\mathcal{N})}=\alpha \int d^{3} y\left[\hat{\rho}^{(\mathcal{N})}(\boldsymbol{y}) \phi^{(\mathrm{ext})}(\boldsymbol{y})-\hat{\boldsymbol{j}}^{(\mathcal{N})}(\boldsymbol{y}) \cdot \boldsymbol{A}^{(\mathrm{ext})}(\boldsymbol{y})\right]
$$

where the hat over the nuclear potentials indicates that they are operators rather than $c$-number functions. Again, the nuclear charge and three-current operators include a variety of terms that can contribute to PCTC and PVTV interactions. A multipole expansion is helpful in separating the PCTC and PVTV terms.

\section{The spherical multipole expansion}

To divide $H_{\text {int }}^{(e \mathcal{N})}$ and $H_{\mathrm{ext}}^{(\mathcal{N})}$ into PCTC and PVTV interactions, we decompose the potentials,

$$
\begin{gathered}
\phi^{(\mathcal{N})}(\boldsymbol{x})=\int d^{3} y \frac{\hat{\rho}^{(\mathcal{N})}(\boldsymbol{y})}{|\boldsymbol{x}-\boldsymbol{y}|}, \\
\boldsymbol{\alpha} \cdot \boldsymbol{A}^{(\mathcal{N})}(\boldsymbol{x})=\int d^{3} y \frac{\boldsymbol{\alpha} \cdot \hat{\boldsymbol{j}}^{(\mathcal{N})}(\boldsymbol{y})}{|\boldsymbol{x}-\boldsymbol{y}|},
\end{gathered}
$$

into multipoles. One expands the photon Green's function in terms of spherical harmonics $Y_{J}^{M}$,

$$
\begin{aligned}
\frac{1}{|\boldsymbol{x}-\boldsymbol{y}|}= & \sum_{J \geqslant 0} \frac{4 \pi}{2 J+1}\left[\theta(x-y) \frac{y^{J}}{x^{J+1}}+\theta(y-x) \frac{x^{J}}{y^{J+1}}\right] \\
& \times Y_{J}(\hat{x}) \odot Y_{J}(\hat{y}),
\end{aligned}
$$

where

$$
Y_{J}(\hat{x}) \odot Y_{J}(\hat{y}) \equiv \sum_{M} Y_{J}^{M *}(\hat{x}) Y_{J}^{M}(\hat{y}) .
$$

The resulting expressions for the potentials are

$$
\begin{aligned}
\phi^{(\mathcal{N})}(\boldsymbol{x}) & =\int d^{3} y \frac{\hat{\rho}^{(\mathcal{N})}(\boldsymbol{y})}{|\boldsymbol{x}-\boldsymbol{y}|} \\
& =\sum_{J \geqslant 0} \frac{4 \pi}{2 J+1} \frac{1}{x^{J+1}} Y_{J}(\hat{x}) \odot\left[\hat{C}_{J}+\hat{\mathcal{C}}_{J}(x)\right],
\end{aligned}
$$




$$
\begin{aligned}
\boldsymbol{\alpha} \cdot \boldsymbol{A}^{(\mathcal{N})}(\boldsymbol{x})= & \int d^{3} y \frac{\boldsymbol{\alpha} \cdot \hat{\boldsymbol{j}}^{(\mathcal{N})}(\boldsymbol{y})}{|\boldsymbol{x}-\boldsymbol{y}|} \\
= & -\sum_{J \geqslant 1} \frac{4 \pi}{2 J+1} \frac{1}{x^{J+1}}\left[Y_{J}(\hat{x}) \otimes \boldsymbol{\alpha}\right]_{J} \\
& \odot\left[\hat{M}_{J}+\hat{\mathcal{M}}_{J}(x)\right]+\cdots .
\end{aligned}
$$

Here $[A \otimes B]_{J}^{M}$ is the standard notation for coupling two spherical tensors $A$ and $B$ to a tensor of rank $J$, and the " $+\cdots "$ indicates the contributions from the transverse electric multipoles, which do not contribute to either PCTC or PVTV moments for their $\mathrm{P}$ and $\mathrm{T}$ transformation properties. ${ }^{4}$ And we note that the nuclear vector potential $\boldsymbol{A}^{(\mathcal{N})}$ satisfies the Coulomb gauge.

The charge $(\hat{C}$ and $\hat{\mathcal{C}})$ and transverse magnetic $(\hat{M}$ and $\hat{\mathcal{M}})$ nuclear multipole operators are defined as

$$
\begin{aligned}
\hat{C}_{J}^{M} & =\int d^{3} y y^{J} Y_{J}^{M}(\hat{y}) \hat{\rho}(\boldsymbol{y}), \\
\hat{M}_{J}^{M} & =\int d^{3} y\left[y^{J} Y_{J}(\hat{y}) \otimes \hat{\boldsymbol{j}}(\boldsymbol{y})\right]_{J}^{M}, \\
\hat{\mathcal{C}}_{J}^{M}(x) & =\int d^{3} y \theta(y-x)\left[(x / y)^{2 J+1}-1\right] y^{J} Y_{J}^{M}(\hat{y}) \hat{\rho}(\boldsymbol{y}) \\
& \equiv \hat{\mathcal{C}}_{J<}^{M}(x)-\hat{\mathcal{C}}_{J>}^{M}(x), \\
\hat{\mathcal{M}}_{J}^{M}(x) & =\int d^{3} y \theta(y-x)\left[(x / y)^{2 J+1}-1\right]\left[y^{J} Y_{J}(\hat{y}) \otimes \hat{j}(\boldsymbol{y})\right]_{J}^{M} \\
& \equiv \hat{\mathcal{M}}_{J<}^{M}(x)-\hat{\mathcal{M}}_{J>}^{M}(x) .
\end{aligned}
$$

In the definitions of $\hat{\mathcal{C}}(x)$ and $\hat{\mathcal{M}}(x)$ - the penetration terms that account for contributions when the electron cloud is inside the nucleus-the subscripts " $<$ " and " $>$ " refer to the parts containing $(x / y)^{2 J+1}$ and 1 , respectively.

The moments (diagonal matrix elements) of multipole operators in Eqs. (16)-(18d) have definite parity and timereversal characteristics depending on their angular momentum, as indicated in Table I. For completeness, we have also included the moments of the transverse electric multipole operators, $\hat{E}_{J}^{M}$ and $\hat{\mathcal{E}}_{J}^{M}(x)$. These are not relevant in PVTV calculations in which only nuclear moments are considered. A PVTV nuclear moment can arise if and only if some source of parity and time-reversal violation is introduced. Thus, nonzero

\footnotetext{
${ }^{4}$ For example, the $J=1$ transverse electric multipole moment gives the nuclear anapole moment [28].
}

TABLE I. Parity and time-reversal characteristics of the Coulomb $\left(C_{J}^{M}\right)$, magnetic $\left(M_{J}^{M}\right)$, and transverse electric $\left(E_{J}^{M}\right)$ multipole moments. $\mathrm{x}$ indicates no contribution transforming as indicated.

\begin{tabular}{lcccc}
\hline \hline $\begin{array}{l}\text { Multipole } \\
\text { moment }\end{array}$ & PCTC & PVTV & PVTC & PCTV \\
\hline$C_{J}^{M}, \mathcal{C}_{J}^{M}(x)$ & even $J$ & odd $J$ & x & x \\
$M_{J}^{M}, \mathcal{M}_{J}^{M}(x)$ & odd $J$ & even $J$ & x & x \\
$E_{J}^{M}, \mathcal{E}_{J}^{M}(x)$ & x & x & odd $J$ & even $J$ \\
\hline \hline
\end{tabular}

moments corresponding to odd charge multipoles or even magnetic multipoles are the signatures of PVTV interactions.

The electron-nucleus interaction can be divided formally into PCTC and PVTV components:

$$
\begin{aligned}
H_{\mathrm{int}}^{(e \mathcal{N})} & =-\alpha \sum_{i=1}^{Z}\left[\phi_{i}^{(\mathcal{N})}-\boldsymbol{\alpha}_{i} \cdot \boldsymbol{A}_{i}^{(\mathcal{N})}\right]+\tilde{V}_{\mathrm{int}}^{(\tilde{e} \mathcal{N})} \\
& \equiv V_{\mathrm{int}}^{(e \mathcal{N})}+\tilde{V}_{\mathrm{int}}^{(e \tilde{\mathcal{N}})}+\tilde{V}_{\mathrm{int}}^{(\tilde{e} \mathcal{N})}
\end{aligned}
$$

where $\tilde{V}_{\text {int }}^{(\tilde{e} \mathcal{N})}$ was defined in Eq. (7). In general the multipole decomposition is helpful in separating the first two terms. For example, in the case most often of interest where a nuclear moment is taken,

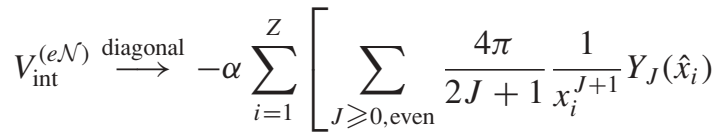

$$
\begin{aligned}
& \odot\left[\hat{C}_{J}+\hat{C}_{J}\left(x_{i}\right)\right]+\sum_{J \geqslant 1, \text { odd }} \frac{4 \pi}{2 J+1} \frac{1}{x_{i}^{J+1}} \\
& \times\left[Y_{J}\left(\hat{x}_{i}\right) \otimes \boldsymbol{\alpha}\right]_{J} \odot\left[\hat{M}_{J}+\hat{\mathcal{M}}_{J}\left(x_{i}\right)\right] \text {, } \\
& V_{\text {int }}^{(e \tilde{\mathcal{N}})} \stackrel{\text { diagonal }}{\longrightarrow}-\alpha \sum_{i=1}^{Z}\left[\sum_{J \geqslant 1, \text { odd }} \frac{4 \pi}{2 J+1} \frac{1}{x_{i}^{J+1}} Y_{J}\left(\hat{x}_{i}\right)\right. \\
& \odot\left[\hat{C}_{J}+\hat{\mathcal{C}}_{J}\left(x_{i}\right)\right]+\sum_{J \geqslant 2, \text { even }} \frac{4 \pi}{2 J+1} \frac{1}{x_{i}^{J+1}} \\
& \left.\times\left[Y_{J}\left(\hat{x}_{i}\right) \otimes \boldsymbol{\alpha}\right]_{J} \odot\left[\hat{M}_{J}+\hat{\mathcal{M}}_{J}\left(x_{i}\right)\right]\right] \text {. }
\end{aligned}
$$

Thus one can identify the total electron-nucleus PVTV interaction $\tilde{V}_{\text {int }}^{(e \tilde{\mathcal{N}}+\tilde{e} \mathcal{N})}$, that is, the sum of terms where the PVTV coupling is either on the nucleus or on the electrons:

$$
\tilde{V}_{\text {int }}^{(e \tilde{\mathcal{N}}+\tilde{e} \mathcal{N})}=\tilde{V}_{\text {int }}^{(e \tilde{\mathcal{N}})}+\tilde{V}_{\text {int }}^{(\tilde{e} \mathcal{N})}
$$

In connection with Eq. (21), it might be helpful at this point to comment about nuclear sources of PVTV interactions, which can originate from direct PVTV electromagnetic couplings to a nucleon (i.e., one-body PVTV currents generated by nucleon EDMs), couplings to various meson exchanges or to $N \bar{N}$ excitations (two-body PVTV currents), or PVTV interactions of any type between nucleons. The last would give rise to small PVTV admixtures in nuclear wave functions. Thus we see, working to first order in PVTV, that there are two classes of contributions to PVTV nuclear moments (see, e.g., Refs. [29,30] and references therein):

(i) The PVTV current contribution: Small components of the multipole operators resulting from PVTV contributions to one- or two-body charges or threecurrents-operators that might be denoted as $\hat{\tilde{C}}_{J=\text { odd }}$ and $\hat{\tilde{M}}_{J=\text { even }}$-would have nonzero matrix elements between the dominant PCTC component of the nuclear wave function. 


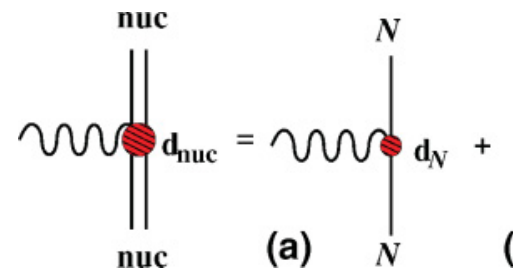

(b)
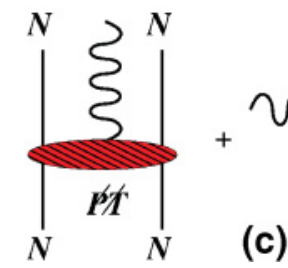

(ii) The PVTV nuclear polarization contribution: Large components of the multipole operators resulting from the ordinary PCTC currents would have nonzero matrix elements connecting PCTC components in the bra state with PVTV "polarization" admixtures in the ket state, and vice versa.

These contributions to the nuclear EDM are illustrated in Fig. 1.

In the multipole expressions, Eqs. (16)-(21), we have been careful to isolate terms associated with the finite nuclear size (and thus electron penetration) from those that would persist in the point-nucleus limit. Nuclear matrix elements of the operators $\hat{C}_{J}$ and $\hat{M}_{J}$ correspond to the standard static moments [31] defined by Taylor expanding the Green's function around $\boldsymbol{y}=0$, a procedure that implicitly assumes $|\boldsymbol{x}| \gg|\boldsymbol{y}|$. As indicated by Eq. (14), the complete expansion of the Green's function contains terms corresponding to both $|\boldsymbol{x}|>|\boldsymbol{y}|$ as well as $|\boldsymbol{y}|>|\boldsymbol{x}|$. Thus, to express the potentials in terms of the static nuclear moments, we have used $\theta(x-y)=1-\theta(y-x)$ and collected all terms proportional to $\theta(y-x)$ in the "local" multipole operators $\hat{\mathcal{C}}_{J}(x)$ and $\hat{\mathcal{M}}_{J}(x) .{ }^{5}$ Physically, the static multipoles characterize the interaction of the electrons with a pointlike nucleus, whereas the local multipoles correspond to the penetration of the electrons inside the nucleus.

The multipole expansion also provides a useful framework for decomposing the interaction of the nuclear charge and current with an external potential. In the present instance we distinguish the terms in $H_{\text {ext }}^{(\mathcal{N})}$ according to the transformation properties of the various multipole components:

$$
H_{\mathrm{ext}}^{(\mathcal{N})}=V_{\mathrm{ext}}^{(\mathcal{N})}+\tilde{V}_{\mathrm{ext}}^{(\tilde{\mathcal{N}})}
$$

where $V_{\text {ext }}^{(\mathcal{N})}$ is even under $\mathrm{P}$ and $\mathrm{T}$ whereas $\tilde{V}_{\text {ext }}^{(\tilde{\mathcal{N}})}$ has the opposite transformation properties. For example, $V_{\text {ext }}^{(\mathcal{N})}$ contains the interaction of the nuclear magnetic dipole moment with the constant applied magnetic field, and $\tilde{V}_{\text {ext }}^{(\tilde{\mathcal{N}})}$ contains the interaction of the nuclear EDM with the constant applied electric field, $\boldsymbol{E}_{0}^{(\mathrm{ext})}$. Substituting the potential for the latter,

$$
\phi^{(\mathrm{ext})}(\boldsymbol{y})=-\alpha \boldsymbol{E}_{0}^{(\mathrm{ext})} \cdot \boldsymbol{y},
$$

into Eq. (11) leads to

$$
\tilde{V}_{\mathrm{ext}}^{(\tilde{\mathcal{N}})}=-\alpha \boldsymbol{d}_{\mathcal{N}} \cdot \boldsymbol{E}_{0}^{(\mathrm{ext})}
$$

${ }^{5}$ A "local" multipole means it can only interact with another field when they overlap. The classical example is the extra $\delta$ function term introduced in the Cartesian multipole expansion [31].
FIG. 1. (Color online) The contributions to a nuclear EDM from (a) nucleon EDMs, (b) PVTV exchange currents, and (c) parity admixtures induced by PVTV $N N$ interactions (polarization contribution).

where $\boldsymbol{d}_{\mathcal{N}}$ is the nuclear EDM given in terms of $\hat{C}_{1}^{M}$ as

$$
\boldsymbol{d}_{\mathcal{N}}=\left(\frac{4 \pi}{3}\right)^{1 / 2} \sum_{M} \hat{C}_{1}^{M} \boldsymbol{e}_{M}^{*}
$$

where $\boldsymbol{e}_{M}$ is the spherical unit vector.

\section{The unperturbed and the perturbed Hamiltonian}

We are now in a position to identify the components of the unperturbed and the perturbed atomic Hamiltonian. The unperturbed Hamiltonian is

$$
H_{0}=\sum_{i=1}^{Z}\left(\beta_{i} m_{e}+\boldsymbol{\alpha}_{i} \cdot \boldsymbol{p}_{i}\right)+V_{\mathrm{int}}^{(e e)}+V_{\mathrm{int}}^{(e \mathcal{N})}+H_{\mathrm{int}}^{\mathrm{nuc}} .
$$

This Hamiltonian contains the following interactions:

(i) The free electron Hamiltonian, $\beta_{i} m_{e}+\boldsymbol{\alpha}_{i} \cdot \boldsymbol{p}_{i}$ : The electrons are treated as relativistic, pointlike particles.

(ii) The PCTC $e$-e interaction, $V_{\text {int }}^{(e e)}:$ As electrons are treated relativistically, both Coulomb and Breit interactions (the latter of which is not electrostatic) are included. In the Coulomb gauge, the scalar and vector potentials acting on the $i$ th electron in Eq. (4) are

$$
\phi_{i}^{(e)}=\sum_{j \neq i} \frac{1}{x_{i j}}, \quad \boldsymbol{A}_{i}^{(e)}=\sum_{j \neq i} \frac{1}{2 x_{i j}}\left(\boldsymbol{\alpha}_{j}+\boldsymbol{x}_{i j} \frac{\boldsymbol{\alpha}_{j} \cdot \boldsymbol{x}_{i j}}{x_{i j}^{2}}\right),
$$

where $x_{i j}=\left|\boldsymbol{x}_{i j}\right| \equiv\left|\boldsymbol{x}_{i}-\boldsymbol{x}_{j}\right|$.

(iii) The PCTC $e-\mathcal{N}$ interaction, $V_{\text {int }}^{(e \mathcal{N})}:$ In atomic physics, the nucleus is typically considered as a stable, external $c$-number source of electromagnetic fields acting on the electrons. Here, however, we take the PCTC nuclear potentials $\phi^{(\mathcal{N})}$ and $\boldsymbol{A}^{(\mathcal{N})}$, Eqs. (12)-(17), to be dynamical quantities and expand them in terms of nuclear multipole moment operators, as described previously. We will later resort to the $c$-number source approximation when considering practical atomic calculations.

(iv) The PCTC internal nuclear Hamiltonian, $H_{\mathrm{int}}^{\text {nuc }}$ : Again, in typical atomic computations, $H_{0}$ is diagonalized by using product wave functions that separately diagonalize $H_{\text {int }}^{\text {nuc }}$ and the electronic operators in Eq. (2). We will not resort to this approximate diagonalization until Sec. III, so we must include $H_{\text {int }}^{\text {nuc }}$ explicitly.

The perturbed Hamiltonian contains PCTC interactions between the electrons and nucleus with the external field (terms with no tildes) and PVTV interactions within the atom or with 


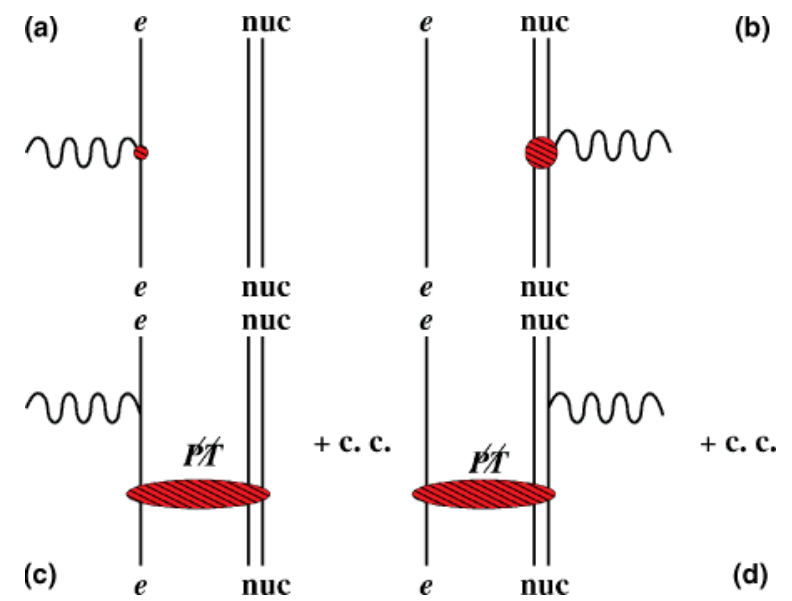

FIG. 2. (Color online) Direct interactions between the external field and (a) electron EDM and (b) nuclear EDM along with external-field-dependent induced terms involving (c) electronic polarization and (d) nuclear polarization that result in Schiff screening and its corrections. Throughout the remainder of this paper, type (d) corrections are not analyzed explicitly.

the external field (terms with tildes):

$$
\begin{aligned}
H_{I}= & V_{\mathrm{ext}}^{(e)}+V_{\mathrm{ext}}^{(\mathcal{N})}+\left[\tilde{V}_{\mathrm{ext}}^{(\tilde{e})}+\tilde{V}_{\mathrm{int}}^{(\tilde{e} \mathcal{N})}+\tilde{V}_{\mathrm{int}}^{(\tilde{e} e)}\right] \\
& +\left[\tilde{V}_{\mathrm{ext}}^{(\tilde{\mathcal{N}})}+\tilde{V}_{\mathrm{int}}^{(e \tilde{\mathcal{N}})}+\tilde{V}_{\mathrm{int}}^{\mathrm{nuc}}\right],
\end{aligned}
$$

where we have collected together the terms proportional to the electron EDM as well as the terms involving PVTV nuclear couplings. The PVTV internal nuclear potential, $\tilde{V}_{\mathrm{int}}^{\text {nuc }}$, is included in the latter as one of the perturbations. Its presence will induce mixing among nuclear states of opposite parity. As already discussed, this mixing leads to nonvanishing matrix elements of the PVTV nuclear moment operators involving the ordinary nuclear charge and current operators. If $\tilde{V}_{\text {int }}^{\text {nuc }}$ carries a momentum dependence, the nuclear continuity equation also requires the presence of exchange charge and three-current operators, $\hat{\tilde{\rho}}(\boldsymbol{y})$ and $\hat{\tilde{j}}(\boldsymbol{y})$, that have the opposite parity and time-reversal transformation properties compared to the ordinary charge and current operators. Insertion of $\hat{\tilde{\rho}}(\boldsymbol{y})$ and $\hat{\tilde{j}}(\boldsymbol{y})$ into the PVTV nuclear multipole operators yields operators that have nonvanishing matrix elements between same-parity states.

\section{B. Schiff screening and its corrections}

The observability of the EDM of a neutral composite system is severely hampered by the screening effect, the cancellation between direct interactions of the external field with electron or nuclear EDMs and the terms involving the internal fields induced by the applied field. The terms we will discuss in the next two sections are illustrated in Fig. 2. Compared to (c), (d) not only has a suppression from the higher nuclear excitation energy $\left(\Delta E_{\text {atom }} / \Delta E_{\text {nuc }} \sim 10^{-6}\right)$, but also the $e-\mathcal{N}$ electromagnetic interaction here can only go through higher nuclear multipoles such as $\mathcal{C}_{0}(x)$ and $C_{2}$ or $M_{1}$, which, in combination with the charge dipole transition matrix element, results in an additional finite-size or hyperfine suppression factor, of $\sim 10^{-9}$ or $10^{-7}$, respectively. Therefore, panel (d) can be safely ignored in the subsequent discussion.

As Schiff pointed out in deriving his theorem [11], there exist three types of effects that contradict the assumptions of the Schiff theorem and, thus, make the shielding incomplete: (a) The constituent particles are relativistic; (b) the constituents have finite size; or (c) there exist nonelectrostatic interactions between the constituents. All three effects are present in atomic systems: (a) The atomic electrons may be relativistic, especially for heavy atoms; (b) the atomic nucleus has a finite spatial extent; and (c) the $e-e$ and $e-\mathcal{N}$ electromagnetic interactions contain current-current components that are not electrostatic. In this section, all of these factors will be gathered together, and a more unified and consistent derivation of corrections to Schiff screening effect will be presented.

In the literature one can find several ways of demonstrating that EDMs cause no first-order energy shift, such as representing an EDM as an infinitesimal displaced charge with the help of translational operators as in Schiff's original paper [11], or representing the shielding as a hypervirial theorem with the help of the Hellmann-Feynman theorem as in Ref. [18]. In this paper, we follow the approach taken in Refs. $[13,14]$ and rely on perturbation theory. We concentrate first on corrections to the screening of $d_{e}$ and follow it with a treatment of the more involved case of $d_{\mathcal{N}}$.

\section{Shielding of the electron EDM}

To evaluate the consequences of shielding of an electron EDM, we consider the first- and second-order energy shifts that depend on both $d_{e}$ and $\boldsymbol{E}^{\text {(ext) }}$ :

$$
\begin{aligned}
\Delta E_{(1)}^{(\tilde{e})}= & \left\langle\text { g.s. }\left|\tilde{V}_{\text {ext }}^{(\tilde{e})}\right| \text { g.s. }\right\rangle, \\
\Delta E_{(2)}^{(\tilde{e})}= & \sum_{n} \frac{1}{E_{\text {g.s. }}-E_{n}}\left\{\left\langle\text { g.s. }\left|\tilde{V}_{\text {int }}^{(\tilde{e})}+\tilde{V}_{\text {int }}^{(\tilde{e} \mathcal{N})}\right| n\right\rangle\right. \\
& \left.\times\left\langle n\left|V_{\text {ext }}^{(e)}\right| \text { g.s. }\right\rangle+\text { c.c. }\right\},
\end{aligned}
$$

where $\mid$ g.s. $\rangle$ and $|n\rangle$ denote the unperturbed atomic (electronic plus nuclear) ground and excited states, respectively:

$$
\left.\left.H_{0} \mid \text { g.s. }\right\rangle=E_{\text {g.s. }} \mid \text { g.s }\right\rangle, \quad H_{0}|n\rangle=E_{n}|n\rangle .
$$

The first-order energy shift $\Delta E_{(1)}^{(\tilde{e})}$ arises from the interaction of the external field with the electron EDMs, while $\Delta E_{(2)}^{(\tilde{e})}$ contains the effects from the PCTC excitation of the atomic cloud by the external field and its de-excitation by the PVTV $\tilde{e}-e$ and $\tilde{e}-\mathcal{N}$ interactions (and vice versa).

Following Schiff, it is useful to express the PVTV interaction $\tilde{V}_{\text {int }}^{(\tilde{e} e)}+\tilde{V}_{\text {int }}^{(\tilde{e} \mathcal{N})}$ in terms of an appropriate commutator with 
$H_{0}$ plus correction terms. One has ${ }^{6}$

$$
\begin{aligned}
\tilde{V}_{\mathrm{int}}^{(\tilde{e} e)}+\tilde{V}_{\mathrm{int}}^{(\tilde{e} \mathcal{N})}= & -\sum_{i=1}^{Z}\left[d_{e} \beta \boldsymbol{\sigma}_{i} \cdot \nabla_{i}, H_{0}\right]+2 d_{e} \beta i \gamma_{5} \\
& \times\left[\boldsymbol{p}_{i}^{2}+\alpha\left(\boldsymbol{A}_{i}^{(\mathcal{N})}-\frac{1}{2} \boldsymbol{A}_{i}^{(e)}\right) \cdot \boldsymbol{p}_{i}\right] .
\end{aligned}
$$

In the nonrelativistic limit, the first term on the right-hand side of Eq. (33) contains the commutator of $H_{0}$ with Schiff's displacement operator, $d_{e} \sigma \cdot \nabla$. Letting $H_{0}$ act on either side of the relativistic form of this operator in Eq. (31) leads to the energy difference $E_{\text {g.s. }}-E_{n}$ that cancels the corresponding energy denominator. One may then carry out the closure sum on the states $|n\rangle$, leading to

$$
\begin{aligned}
\Delta E_{(2)}^{(\tilde{e})}= & \sum_{i=1}^{Z}\left\langle\text { g.s. }\left|\left[d_{e} \beta \sigma_{i} \cdot \nabla_{i}, V_{\text {ext }}^{(e)}\right]\right| \text { g.s. }\right\rangle+2 i d_{e} \sum_{n} \frac{1}{E_{\text {g.s. }}-E_{n}} \\
& \times\left\{\left\langle\text { g.s. }\left|\sum_{i=1}^{Z} \beta \gamma_{5}\left[\boldsymbol{p}_{i}^{2}+\alpha\left(\boldsymbol{A}_{i}^{(\mathcal{N})}-\frac{1}{2} \boldsymbol{A}_{i}^{(e)}\right) \cdot \boldsymbol{p}_{i}\right]\right| n\right\rangle\right. \\
& \left.\times\left\langle n\left|V_{\text {ext }}^{(e)}\right| \text { g.s. }\right\rangle+\text { c.c. }\right\} .
\end{aligned}
$$

After performing some Dirac algebra on the first term on the right-hand side of Eq. (34) and adding $\Delta E_{(1)}^{(\tilde{e})}$ and $\Delta E_{(2)}^{(\tilde{e})}$ we obtain

$$
\Delta E_{(1)}^{(\tilde{e})}+\Delta E_{(2)}^{(\tilde{e})}=\Delta E_{\left(1^{\prime}\right)}^{(\widetilde{e})}+\Delta E_{\left(2^{\prime}\right)}^{(\widetilde{e})},
$$

where

$$
\begin{aligned}
\Delta E_{\left(1^{\prime}\right)}^{(\tilde{e})}= & \left\langle\text { g.s. }\left|\sum_{i=1}^{Z} 2 \alpha d_{e} \beta i \gamma_{5} \boldsymbol{A}_{i}^{(\mathrm{ext})} \cdot \boldsymbol{p}_{i}\right| \text { g.s. }\right\rangle \\
\Delta E_{\left(2^{\prime}\right)}^{(\tilde{e})}= & 2 i d_{e} \sum_{n} \frac{1}{E_{\text {g.s. }}-E_{n}}\left\{\langle \text { g.s. } | \sum _ { i = 1 } ^ { Z } \beta \gamma _ { 5 } \left[\boldsymbol{p}_{i}^{2}+\alpha\left(\boldsymbol{A}_{i}^{(\mathcal{N})}\right.\right.\right. \\
& \left.\left.\left.-\frac{1}{2} \boldsymbol{A}_{i}^{(e)}\right) \cdot \boldsymbol{p}_{i}\right]|n\rangle\left\langle n\left|V_{\text {ext }}^{(e)}\right| \text { g.s. }\right\rangle+\text { c.c. }\right\}
\end{aligned}
$$

The effective residual first-order contribution $\Delta E_{\left(1^{\prime}\right)}^{(\widetilde{e})}$ arises from a partial cancellation between $\Delta E_{(1)}^{(\tilde{e})}$ and the commutator term of $\Delta E_{(2)}^{(\tilde{e})}$ in Eq. (34). Both $\Delta E_{\left(1^{\prime}\right)}^{(\tilde{e})}$ and $\Delta E_{\left(2^{\prime}\right)}^{(\widetilde{e})}$ involve matrix elements of $\gamma_{5}$ that connects the large (upper) and the small (lower) component of a Dirac wave function. Thus, for nonrelativistic (NR) electrons (with vanishing lower components), $\Delta E_{\left(1^{\prime}\right)}^{(\widetilde{e})}=\Delta E_{\left(2^{\prime}\right)}^{(\widetilde{e})}=0$, implying complete shielding of electron EDMs.

It should be noted that the procedure of using a closure sum to evaluate the effects of shielding is not unique. The

\footnotetext{
${ }^{6} \mathrm{~A}$ similar commutation relation was also considered by Lindroth et al. [16] and was referred to as "stratagem II." It contrasts with the earlier work by Sandars [13] ("stratagem I"), where a commutation relation without the $\beta$ matrix was employed. The difference between the present work and Ref. [16] is that neither nuclear nor external vector potentials were included in Ref. [16].
}

first discussion of relativistic effects by Sandars [13] relies on expressing the Dirac matrix $\beta$ in the EDM interaction as $1+(\beta-1)$, and using closure just to remove the " 1 " term while leaving the $(\beta-1)$ term intact. This approach leads to a less complete cancellation of $\Delta E_{(1)}^{(\tilde{e})}$. However, as the use of $(\beta-1)$ also requires nonvanishing small components, it has the same NR limit as the $\gamma_{5}$ formalism presented here. In a later publication [16], Lindroth et al. argued that although the $\gamma_{5}$ formalism has the advantage of being purely one-body when no Breit interaction is present, it is not formally of order $Z^{2} \alpha^{2}$, in contrast to the $(\beta-1)$ formalism. This might introduce complications when atomic many-body calculations reach this level of accuracy [16]. However, no detailed calculation further substantiates this claim. In this paper, the $\gamma_{5}$ formalism is chosen for its simplicity.

\section{Shielding of the nuclear EDM}

To evaluate the effects of shielding of a nuclear EDM, we follow a procedure similar to that used in the case of the electron and consider energy shifts that are linear in $\boldsymbol{d}_{\mathcal{N}}$ and $\boldsymbol{E}^{\text {(ext) }}$ to the second order in perturbation theory. However, we depart from the previous notation somewhat in that we make the polarization effect of $\tilde{V}_{\text {int }}^{\text {nuc }}$ explicit in the nuclear wave functions. This yields

$$
\begin{aligned}
\Delta E_{(1)}^{(\tilde{\mathcal{N}})}= & \left\langle\text { g.s. } \tilde{\mathcal{N}}\left|\tilde{V}_{\mathrm{ext}}^{(\tilde{\mathcal{N}})}\right| \mathrm{g} . \mathrm{s} \cdot \tilde{\mathcal{N}}\right\rangle \\
\Delta E_{(2)}^{(\tilde{\mathcal{N}})}= & \sum_{n} \frac{1}{E_{\text {g.s. }}-E_{n}} \\
& \times\left\{\left\langle\text { g.s. }\left|V_{\text {ext }}^{(e)}\right| n\right\rangle\left\langle n_{\tilde{\mathcal{N}}}\left|\tilde{V}_{\text {int }}^{(e \tilde{\mathcal{N}})}\right| \text { g.s. } \tilde{\mathcal{N}}\right\rangle+\text { c.c. }\right\} .
\end{aligned}
$$

For example, if the atomic wave function has the direct-product form $|n\rangle=|n\rangle_{\mathcal{N}} \otimes|n\rangle_{e}$, then

$$
\begin{aligned}
\left|n_{\tilde{\mathcal{N}}}\right\rangle= & \sum_{m} \frac{1}{E_{n_{\mathcal{N}}}-E_{m_{\mathcal{N}}}} \\
& \times_{\mathcal{N}}\left\langle m\left|\tilde{V}_{\text {int }}^{\text {nuc }}\right| n\right\rangle_{\mathcal{N}}|m\rangle_{\mathcal{N}} \otimes|n\rangle_{e} \equiv|\tilde{n}\rangle_{\mathcal{N}} \otimes|n\rangle_{e} .
\end{aligned}
$$

It is understood that PVTV effects are to be evaluated only in first order. Thus, the meaning of the notation in Eqs. (38) and (39) is as follows:

(i) Contributions involving PVTV charges or threecurrents in $\tilde{V}_{\text {ext }}^{(\tilde{\mathcal{N}})}$ or $\tilde{V}_{\text {int }}^{(e \tilde{\mathcal{N}})}$ are to be evaluated with unperturbed wave functions.

(ii) Contributions involving $\tilde{V}_{\text {int }}^{\text {nuc }}$ correspond to matrix elements with PCTC charges or currents in $\tilde{V}_{\text {ext }}^{(\tilde{\mathcal{N}})}$ or $\tilde{V}_{\text {int }}^{(e \tilde{\mathcal{N}})}$. Furthermore, $\tilde{V}_{\text {int }}^{\text {nuc }}$ is to be treated in first order, modifying the bra or the ket state, but not both.

In the following we suppress the explicit subscript $\tilde{\mathcal{N}}$ on bra and ket states involving operators coupling to the nucleus, but implicitly $\tilde{V}_{\text {int }}^{\text {nuc }}$ has been absorbed into nuclear wave functions and its effects will be retained to first order in the PVTV. 
To proceed, we first write $\boldsymbol{d}_{\mathcal{N}}$ in a spherical basis using Eq. (26) and observe that

$$
\left[\boldsymbol{d}_{\mathcal{N}} \cdot \nabla, \frac{1}{x}\right]=-\left(\frac{4 \pi}{3}\right) \hat{C}_{1} \otimes Y_{1}\left(\Omega_{x}\right) \frac{1}{x^{2}} .
$$

Letting

$$
\Delta H_{0} \equiv H_{0}-H_{0}^{e-\mathcal{N}}\left(C_{0} \text { only }\right)=H_{0}+Z \alpha \sum_{i=1}^{Z} \frac{1}{x_{i}}
$$

be the unperturbed Hamiltonian without the static electronnucleus PCTC monopole Coulomb interaction, we have that

$$
\begin{aligned}
-\alpha \tilde{\phi}_{i}^{\left(C_{1}\right)} & =-\alpha\left(\frac{4 \pi}{3}\right) \hat{C}_{1} \odot \sum_{i=1}^{Z} Y_{1}\left(\Omega_{x_{i}}\right) \frac{1}{x_{i}^{2}} \\
& =\alpha \sum_{i=1}^{Z}\left[\boldsymbol{d}_{\mathcal{N}} \cdot \nabla_{i}, \sum_{j=1}^{Z} \frac{1}{x_{j}}\right] \\
& =-\sum_{i=1}^{Z}\left[\frac{\boldsymbol{d}_{\mathcal{N}}}{Z} \cdot \nabla_{i}, H_{0}-\Delta H_{0}\right] .
\end{aligned}
$$

Substituting Eq. (43) into $\Delta E_{(2)}^{(\tilde{\mathcal{N}})}$ and letting $H_{0}$ act on either side of the displacement operator leads to the energy difference $E_{\text {g.s. }}-E_{n}$ that cancels the energy denominator and allows us to carry out a closure sum for this term. Doing so and letting

$$
\Delta \tilde{V}_{\mathrm{int}}^{(e \tilde{\mathcal{N}})}=\tilde{V}_{\mathrm{int}}^{(e \tilde{\mathcal{N}})}+\alpha \tilde{\phi}_{i}^{\left(C_{1}\right)}
$$

leads to

$$
\begin{aligned}
\Delta E_{(2)}^{(\tilde{\mathcal{N}})}= & \sum_{n} \frac{1}{E_{\text {g.s. }}-E_{n}}\left\{\left\langle\text { g.s. }\left|V_{\mathrm{ext}}^{(e)}\right| n\right\rangle\langle n|-\alpha \tilde{\phi}_{i}^{\left(C_{1}\right)}\right. \\
& \left.\left.+\Delta \tilde{V}_{\mathrm{int}}^{(e \tilde{\mathcal{N}})} \mid \text { g.s. }\right\rangle+ \text { c.c. }\right\} \\
= & \frac{1}{Z}\left\langle\text { g.s. }\left|\left[\sum_{i=1}^{Z} \boldsymbol{d}_{\mathcal{N}} \cdot \nabla_{i}, V_{\mathrm{ext}}^{(e)}\right]\right| \text { g.s. }\right\rangle \\
& +\sum_{n} \frac{1}{E_{\mathrm{g} . \mathrm{s} .}-E_{n}}\left\{\left\langle\text { g.s. }\left|V_{\mathrm{ext}}^{(e)}\right| n\right\rangle\langle n| \Delta \tilde{V}_{\mathrm{int}}^{(e \tilde{\mathcal{N}})}\right. \\
& \left.\left.+\sum_{i=1}^{Z}\left[\frac{\boldsymbol{d}_{\mathcal{N}}}{Z} \cdot \nabla_{i}, \Delta H_{0}\right] \mid \text { g.s. }\right\rangle+ \text { c.c. }\right\} .
\end{aligned}
$$

Now observe that

$$
\begin{aligned}
& \frac{1}{Z}\left[\sum_{i=1}^{Z} \boldsymbol{d}_{\mathcal{N}} \cdot \nabla_{i}, V_{\mathrm{ext}}^{(e)}\right] \\
& \quad=-\frac{\alpha}{Z} \sum_{i, j=1}^{Z}\left[\boldsymbol{d}_{\mathcal{N}} \cdot \nabla_{i}, \phi_{j}^{(\mathrm{ext})}-\boldsymbol{\alpha}_{j} \cdot \boldsymbol{A}_{j}^{(\mathrm{ext})}\right] \\
& \quad=\alpha \boldsymbol{d}_{\mathcal{N}} \cdot \boldsymbol{E}_{0}^{(\mathrm{ext})}+\frac{\alpha}{Z} \sum_{i=1}^{Z}\left[\boldsymbol{d}_{\mathcal{N}} \cdot \nabla_{i}, \boldsymbol{\alpha}_{i} \cdot \boldsymbol{A}_{i}^{(\mathrm{ext})}\right]
\end{aligned}
$$

where we have used the electrostatic stability condition $Z \boldsymbol{E}_{0}^{(\mathrm{ext})}-\sum_{i} \boldsymbol{E}_{i}^{(\mathrm{ext})}=0$. Adding the first- and second-order energy shifts leads to

$$
\begin{aligned}
\Delta E_{(1)}^{(\tilde{\mathcal{N}})}+\Delta E_{(2)}^{(\tilde{\mathcal{N}})}= & \frac{\alpha}{Z}\left\langle\text { g.s. }\left|\sum_{i=1}^{Z}\left[\boldsymbol{d}_{\mathcal{N}} \cdot \nabla_{i}, \boldsymbol{\alpha}_{i} \cdot \boldsymbol{A}_{i}^{(\mathrm{ext})}\right]\right| \mathrm{g} . \mathrm{s} .\right\rangle \\
& +\sum_{n} \frac{1}{E_{\text {g.s. }}-E_{n}}\left\{\left\langle\text { g.s. }\left|V_{\mathrm{ext}}^{(e)}\right| n\right\rangle\langle n| \Delta \tilde{V}_{\mathrm{int}}^{(e \tilde{\mathcal{N}})}\right. \\
& \left.\left.+\sum_{i=1}^{Z}\left[\frac{\boldsymbol{d}_{\mathcal{N}}}{Z} \cdot \nabla_{i}, \Delta H_{0}\right] \mid \text { g.s. }\right\rangle+ \text { c.c. }\right\} .
\end{aligned}
$$

Equation (47) demonstrates the cancellation of all terms proportional to $\boldsymbol{d}_{\mathcal{N}} \cdot \boldsymbol{E}_{0}^{(\mathrm{ext})}$, i.e., the screening effect, and allows us to identify systematically all corrections to this screening. We elaborate on these corrections, including the correction associated with the Schiff moment, in the following.

\section{E. Shielding corrections}

From Eqs. (36), (37), and (47), one may determine all the corrections to Schiff screening that are linear in $d_{e}$ or $d_{\mathcal{N}}$ and that occur to the second order in perturbation theory. For purposes of future discussion, it is useful to classify the corrections as ground-state matrix elements and internal excitations.

\section{Ground-state matrix elements}

The following corrections arise solely from the interaction with the external vector potential $\boldsymbol{A}_{i}^{(\mathrm{ext})}$ :

$$
\Delta E_{(1)}=\left\langle\text { g.s. }\left|\hat{O}_{\mathrm{ext}}^{(\tilde{e})}+\hat{O}_{\mathrm{ext}}^{(\tilde{\mathcal{N}})}\right| \mathrm{g} . \mathrm{s} .\right\rangle,
$$

where

$$
\begin{aligned}
\hat{O}_{\mathrm{ext}}^{(\tilde{e})} & =\sum_{i=1}^{Z} 2 \alpha d_{e} \beta i \gamma_{5} \boldsymbol{A}_{i}^{(\mathrm{ext})} \cdot \boldsymbol{p}_{i}, \\
\hat{O}_{\mathrm{ext}}^{(\tilde{\mathcal{N}})} & =\frac{\alpha}{Z} \sum_{i=1}^{Z}\left[\boldsymbol{d}_{\mathcal{N}} \cdot \nabla_{i}, \boldsymbol{\alpha}_{i} \cdot \boldsymbol{A}_{i}^{(\mathrm{ext})}\right] .
\end{aligned}
$$

\section{Internal excitations}

The remaining corrections involve excitation of atomic states by $V_{\mathrm{ext}}^{(e)}$ and de-excitation by operators proportional to $d_{e}, d_{\mathcal{N}}$, or higher PVTV nuclear moments. The corresponding energy shift is

$$
\begin{aligned}
\Delta E_{(2)}= & \sum_{n} \frac{1}{E_{\text {g.s. }}-E_{n}}\left\{\left\langle\text { g.s. }\left|V_{\mathrm{ext}}^{(e)}\right| n\right\rangle\langle n| \hat{O}_{\mathrm{int}}^{(\tilde{e})}\right. \\
& \left.\left.+\sum_{i=1}^{Z} \sum_{k=1}^{9} \hat{O}_{k, i}^{(\tilde{\mathcal{N}})} \mid \text { g.s. }\right\rangle+ \text { c.c. }\right\},
\end{aligned}
$$

where

$$
\hat{O}_{\text {int }}^{(\tilde{e})}=2 i d_{e} \sum_{i=1}^{Z} \beta \gamma_{5}\left[\boldsymbol{p}_{i}^{2}+\alpha\left(\boldsymbol{A}_{i}^{(\mathcal{N})}-\frac{1}{2} \boldsymbol{A}_{i}^{(e)}\right) \cdot \boldsymbol{p}_{i}\right]
$$




$$
\begin{aligned}
& \hat{O}_{1}^{(\tilde{\mathcal{N}})}=\frac{4 \pi \alpha}{Z} \sum_{J \geqslant 2}^{\text {even }} \frac{1}{x^{J+2}}\left\{\sqrt{\frac{J+1}{2 J+3}} Y_{J+1}(\hat{x}) \odot C_{J}\right.
\end{aligned}
$$

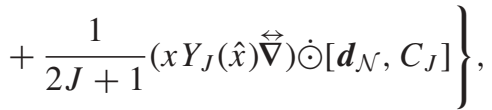

$$
\begin{aligned}
& \hat{O}_{7}^{(\tilde{\mathcal{N}})}=-\sum_{J \geqslant 1}^{\text {odd }} \frac{4 \pi \alpha}{x^{J+1}} \frac{1}{2 J+1} Y_{J}(\hat{x}) \odot \mathcal{C}_{J}(x), \\
& \hat{O}_{8}^{(\tilde{\mathcal{N}})}=-\sum_{J \geqslant 2}^{\text {even }} \frac{4 \pi \alpha}{x^{J+1}} \frac{1}{2 J+1}\left[Y_{J}(\hat{x}) \otimes \alpha\right]_{J} \odot M_{J}, \\
& \hat{O}_{9}^{(\tilde{\mathcal{N}})}=-\sum_{J \geqslant 2}^{\text {even }} \frac{4 \pi \alpha}{x^{J+1}} \frac{1}{2 J+1}\left[Y_{J}(\hat{x}) \otimes \alpha\right]_{J} \odot \mathcal{M}_{J}(x) .
\end{aligned}
$$$$
\hat{O}_{2}^{(\tilde{\mathcal{N}})}=\frac{4 \pi \alpha}{Z} \sum_{J \geqslant 2}^{\text {even }} \frac{1}{x^{J+2}}\left\{\sqrt{\frac{J}{2 J-1}} Y_{J-1}(\hat{x}) \odot\left[\boldsymbol{d}_{\mathcal{N}} \otimes \mathcal{C}_{J<}(x)\right]_{J-1}\right.
$$$$
-\sqrt{\frac{J+1}{2 J+3}} Y_{J+1}(\hat{x}) \odot\left[\boldsymbol{d}_{\mathcal{N}} \otimes \mathcal{C}_{J>}(x)\right]_{J+1}
$$$$
\left.+\frac{1}{2 J+1}\left(x Y_{J}(\hat{x}) \stackrel{\leftrightarrow}{\nabla}\right) \dot{\odot}\left[\boldsymbol{d}_{\mathcal{N}}, \mathcal{C}_{J}(x)\right]\right\}
$$$$
-\frac{4 \pi \alpha}{Z x^{2}} \sqrt{\frac{1}{3}} Y_{1}(\hat{x}) \odot\left[\boldsymbol{d}_{\mathcal{N}} \otimes \mathcal{C}_{0>}(x)\right]_{1}
$$

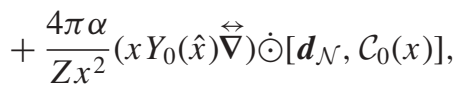$$
\hat{O}_{3}^{(\tilde{\mathcal{N}})}=\frac{4 \pi \alpha}{Z} \sum_{J \geqslant 1}^{\text {odd }} \frac{1}{x^{J+2}}\left\{-\sqrt{\frac{1}{(J+1)(2 J+1)}}\right.
$$$$
\times\left[Y_{J+1}(\hat{x}) \otimes \boldsymbol{\alpha}\right]_{J} \odot\left[\boldsymbol{d}_{\mathcal{N}} \otimes M_{J}\right]_{J}
$$$$
+\sqrt{\frac{J(J+2)}{(J+1)(2 J+3)}}\left[Y_{J+1}(\hat{x}) \otimes \alpha\right]_{J+1}
$$$$
\odot\left[\boldsymbol{d}_{\mathcal{N}} \otimes M_{J}\right]_{J+1}-\frac{1}{2 J+1}\left(x\left[Y_{J}(\hat{x}) \otimes \boldsymbol{\alpha}\right]_{J} \overleftrightarrow{\nabla}\right)
$$$$
\left.\dot{\odot}\left[\boldsymbol{d}_{\mathcal{N}}, M_{J}\right]\right\},
$$$$
\hat{O}_{4}^{(\tilde{\mathcal{N}})}=\frac{4 \pi \alpha}{Z} \sum_{J \geqslant 1}^{\text {odd }} \frac{1}{x^{J+2}}\left\{\sqrt{\frac{1}{(J+1)(2 J+1)}}\left[Y_{J+1}(\hat{x}) \otimes \alpha\right]_{J}\right.
$$$$
\odot\left[\boldsymbol{d}_{\mathcal{N}} \otimes \mathcal{M}_{J>}(x)\right]_{J}
$$$$
+\sqrt{\frac{J(J+2)}{(J+1)(2 J+3)}}\left[Y_{J+1}(\hat{x}) \otimes \boldsymbol{\alpha}\right]_{J+1} \odot\left[\boldsymbol{d}_{\mathcal{N}}\right.
$$$$
\left.\otimes \mathcal{M}_{J>}(x)\right]_{J+1}+\sqrt{\frac{(J+1)(J+3)}{(J+2)(2 J+3)}}\left[Y_{J+1}(\hat{x})\right.
$$$$
\otimes \boldsymbol{\alpha}]_{J+1} \odot\left[\boldsymbol{d}_{\mathcal{N}} \otimes \mathcal{M}_{J<}(x)\right]_{J+1}+\sqrt{\frac{1}{J(2 J+1)}}
$$$$
\times\left[Y_{J-1}(\hat{x}) \otimes \boldsymbol{\alpha}\right]_{J} \odot\left[\boldsymbol{d}_{\mathcal{N}} \otimes \mathcal{M}_{J<}(x)\right]_{J}-\frac{1}{2 J+1}
$$$$
\left.\times\left(x\left[Y_{J}(\hat{x}) \otimes \boldsymbol{\alpha}\right]_{J} \overleftrightarrow{\nabla}\right) \odot\left[\boldsymbol{d}_{\mathcal{N}}, \mathcal{M}_{J}(x)\right]\right\},
$$$$
\hat{O}_{5}^{(\tilde{\mathcal{N}})}=\left[\frac{\boldsymbol{d}_{\mathcal{N}}}{Z} \cdot \overleftrightarrow{\nabla}, H_{\mathrm{int}}^{\mathrm{nuc}}\right]
$$$$
\hat{O}_{6}^{(\tilde{\mathcal{N}})}=-\sum_{J \geqslant 3}^{\text {odd }} \frac{4 \pi \alpha}{x^{J+1}} \frac{1}{2 J+1} Y_{J}(\hat{x}) \odot C_{J},
$$

We refer to Appendix A for more details about the derivation of Eqs. (53)-(61) from Eq. (47) and note important conventions here:

(i) The nuclear composite operators involving $\boldsymbol{d}_{\mathcal{N}}$ and any PCTC nuclear multipole operator $X_{j}$ should be realized as

$$
\begin{aligned}
{\left[\boldsymbol{d}_{\mathcal{N}} \otimes X_{j}\right]_{J}^{M} } & =\left[\boldsymbol{d}_{\mathcal{N}} \otimes X_{j}\right]_{J}^{(\mathrm{sym}) M} \\
& \equiv \sum_{\lambda, m}\langle 1 \lambda, j m \mid J M\rangle\left\{\boldsymbol{d}_{\mathcal{N}}^{\lambda}, X_{j}^{m}\right\} / 2 .
\end{aligned}
$$

As two nuclear density operators do not necessarily commute, the part that does not commute is treated explicitly in the terms involving the commutator of $\boldsymbol{d}_{\mathcal{N}}$ with other nuclear operators in Eqs. (53)-(61).

(ii) The special gradient operator $\stackrel{\leftrightarrow}{\nabla}$ only acts on the electronic bra and ket states as

$$
\begin{aligned}
& { }_{e}\langle|\mathcal{O}(\boldsymbol{x}) \overleftrightarrow{\nabla}|\rangle_{e} \equiv{ }_{e}\langle|\{\mathcal{O}(\boldsymbol{x}), \nabla\}|\rangle_{e} / 2 \\
& \quad=\left({ }_{e}\langle|\mathcal{O}(\boldsymbol{x}) \vec{\nabla}|\rangle_{e}-{ }_{e}\langle|\overleftarrow{\nabla} \mathcal{O}(\boldsymbol{x})|\rangle_{e}\right) / 2,
\end{aligned}
$$

where $\mathcal{O}(\boldsymbol{x})$ denotes a generic operator that depends on electronic degrees of freedom and where in the second equality we have performed an integration by parts.

(iii) The operator " $\odot$ " denotes a double scalar product as

$$
\begin{aligned}
& \left(\mathcal{O}_{J}(\boldsymbol{x}) \overleftrightarrow{\nabla}\right) \odot\left[\boldsymbol{d}_{\mathcal{N}}, X_{J}\right] \\
& \quad=\sum_{\lambda, M}\left(\mathcal{O}_{J}^{M *}(\boldsymbol{x}) \overleftrightarrow{\nabla}^{\lambda *}\right)\left[\boldsymbol{d}_{\mathcal{N}}^{\lambda}, X_{J}^{M}\right]
\end{aligned}
$$

We make several observations about the list of operators $\hat{O}_{k}^{(\tilde{\mathcal{N}})}$.

(i) The operators $\hat{O}_{1-5}^{(\tilde{\mathcal{N}})}$ are generated by the commutator of the displacement operator $\boldsymbol{d}_{\mathcal{N}} \cdot \nabla$ with the non- $C_{0}$ part of the unperturbed Hamiltonian, $\Delta H_{0}$.

(ii) Physically, operators $\hat{O}_{1,3}^{(\tilde{\mathcal{N}})}$ correspond to "displacement" of the static PCTC Coulomb and magnetic electron-nucleus interactions resulting from the rearrangement of the atomic electrons needed to maintain electrostatic equilibrium in the presence of the external field. The operators $\hat{O}_{2,4}^{(\tilde{\mathcal{N}})}$ describe the corresponding effects of displacing the PCTC penetration (nonstatic) $e-\mathcal{N}$ multipole interactions.

(iii) Operator $\hat{O}_{5}^{(\tilde{\mathcal{N}})}$ describes the response of the internal nuclear degrees of freedom to the external field, again as needed to maintain electrostatic equilibrium.

(iv) Operators $\hat{O}_{6-9}^{(\tilde{\mathcal{N}})}$ characterize the effects of the "local" EDM (i.e., $\left.\mathcal{C}_{1}\right)$, PVTV magnetic and higher $(J \geqslant 3)$ 
PVTV charge multipole interactions, including both the static multipole interactions, $\hat{O}_{6,8}^{(\tilde{\mathcal{N}})}$, and penetration terms, $\hat{O}_{7,9}^{(\tilde{\mathcal{N}})}$.

(v) The leading, nonmagnetic correction to Schiff screening resulting from the finite spatial extent and internal structure of the nucleus is the Schiff moment. It therefore arises from the penetration or local multipoles $\mathcal{C}_{J>,<}(x)$ that appear with a $Y_{1}(\hat{x})$; a gradient acting on the electronic coordinates coupled to a $Y_{0}(\hat{x})$ or $Y_{2}(\hat{x})$; and the internal nuclear interaction in conjunction with a gradient acting on the electronic coordinates. Such terms appear in $\hat{O}_{1,2,5,7}^{(\tilde{\mathcal{N}})}$. In the following section, we assemble these terms into the Schiff moment operator and compare with the form of the operator previously used in the literature.

\section{THE SCHIFF MOMENT OPERATOR}

To arrive at the Schiff moment operator, we collect all terms in $\hat{O}_{1,2,5,7}^{(\tilde{\mathcal{N}})}$ that are proportional to $Y_{1}(\hat{x})$ or that contain terms with a gradient acting on the atomic coordinate and transform like a spherical vector:

$$
\begin{aligned}
& {\left[\hat{O}_{1,2,5,7}^{(\tilde{\mathcal{N}})}\right]_{Y_{1}}} \\
& =-\frac{4 \pi \alpha}{Z x^{2}}\left\{\sqrt{\frac{1}{3}} Y_{1}(\hat{x}) \odot\left[\boldsymbol{d}_{\mathcal{N}} \otimes\left(\mathcal{C}_{0>}(x)-\frac{\sqrt{2}}{x^{2}} \mathcal{C}_{2<}(x)\right)\right]_{1}\right. \\
& \quad+Z Y_{1}(\hat{x}) \odot \frac{1}{3} \mathcal{C}_{1}(x) \\
& \quad-\left(x Y_{0}(\hat{x}) \stackrel{\nabla}{\nabla}\right) \odot\left[\boldsymbol{d}_{\mathcal{N}}, \mathcal{C}_{0}(x)\right] \\
& \left.\quad-\left(x Y_{2}(\hat{x}) \stackrel{\leftrightarrow}{\nabla}\right) \odot\left[\boldsymbol{d}_{\mathcal{N}}, \frac{1}{5 x^{2}}\left(C_{2}+\mathcal{C}_{2}(x)\right)\right]\right\} \\
& +\left[\frac{\boldsymbol{d}_{\mathcal{N}}}{Z} \cdot \stackrel{\leftrightarrow}{\nabla}, H_{\mathrm{int}}^{\text {nuc }}\right] \equiv \hat{O}_{\text {Schiff }}^{\text {atomic }} .
\end{aligned}
$$

Note that the terms containing the gradient $\nabla$ acting on the electronic coordinate will connect electronic states differing by one unit of orbital angular momentum, as can be seen by performing an integration by parts and letting $\nabla$ act on the initial and final electronic wave functions. Consequently, we include these operators in $\hat{O}_{\text {Schiff }}^{\text {atomic }}$.

The operator $\hat{O}_{\text {Schiff }}^{\text {atomic }}$ generates the leading contributions to an atomic EDM that depend only on the nuclear charge density operator and not on the current density operator. The form given in Eq. (65) is general and could, in principle, be used to compute the atomic EDM generated by P- and T-odd interactions in the nucleus. From Eq. (51), we observe that $\hat{O}_{\text {Schiff }}^{\text {atomic }}$ will induce mixing of opposite-parity states into the atomic ground state, thereby allowing for a shift in the atomic energy when a constant external electric field is applied (i.e., $\left.V_{\mathrm{ext}}^{(e)}\right)$. In practice, the typical atomic ground state is taken to be a direct product of the nuclear and electronic ground states, as in Eq. (1):

$$
\left.\mid \text { g.s. }\rangle=\mid \text { g.s. }\rangle_{\mathcal{N}} \otimes \mid \text { g.s. }\right\rangle_{e} \text {. }
$$

It is particularly interesting to consider systems in which the electronic ground state is an $\mathrm{S}$ state $(L=0)$, which has a comparatively larger penetration probability because of the lack of the centrifugal barrier. Since $\hat{O}_{\text {Schiff }}^{\text {atomic }}$ transforms as a rank-one tensor in the space of electronic coordinates, it will mix P states into the electronic ground state (relevant for many, but not all atomic EDM experiments). Thus, it is useful to derive a form for this operator applicable to this situation. In doing so, we can derive a nuclear Schiff moment operator that corresponds to- - but differs in form from - the Schiff moment operator used elsewhere in the literature. To that end, we begin by considering the first three terms in $\hat{O}_{\text {Schiff }}^{\text {atomic }}$ and compute their S-P electronic matrix elements.

The electronic wave functions for the $\mathrm{S}$ and $\mathrm{P}$ states are

$$
\begin{aligned}
& \psi_{S}(\boldsymbol{x})=\langle x \mid S, m\rangle=u_{S}(x) Y_{0}(\hat{x}) \chi_{1 / 2}^{m}, \\
& \psi_{P}(\boldsymbol{x})=\left\langle x \mid P, \lambda m^{\prime}\right\rangle=u_{P}(x) Y_{1}^{\lambda}(\hat{x}) \chi_{1 / 2}^{m^{\prime}},
\end{aligned}
$$

where $\lambda$ is the magnetic projection of the $\mathrm{P}$ state $(\lambda=0$ for the $\mathrm{S}$ state), and $\chi_{1 / 2}^{m}$ is the spin wave function. We will henceforth neglect the spin degrees of freedom as they are not relevant to the derivation of the effective nuclear operator- $m$ should be a conserved quantum number in this case. To arrive at the effective nuclear operator, we consider the following polynomial expansions of electronic radial wave functions near the origin:

$$
\begin{aligned}
& u_{S}(x)=\sum_{k \geqslant 0} a_{k} x^{k}, \\
& u_{P}(x)=\sum_{k \geqslant 1} b_{k} x^{k} .
\end{aligned}
$$

We now consider the matrix element of $\hat{O}_{\text {Schiff }}^{\text {atomic }}$ appearing in Eq. (65):

$$
\left\langle n\left|\hat{O}_{\text {Schiff }}^{\text {atomic }}\right| \text { g.s. }\right\rangle={ }_{\mathcal{N}}\left\langle\widetilde{\text { g.S. }}\left|\otimes\left\langle P, \lambda\left|\hat{O}_{\text {Schiff }}^{\text {atomic }}\right| S\right\rangle \otimes\right| \widetilde{\text { g.S. }}\right\rangle_{\mathcal{N}},
$$

and note that we use the convention of Eq. (40). Focusing on the first term in $\hat{O}_{\text {Schiff }}^{\text {atomic }}$ that contains $\mathcal{C}_{0>}(x)$ we obtain an electronic matrix element

$$
\begin{aligned}
& \left\langle P, \lambda\left|\hat{O}_{\text {Schiff }}^{\text {atomic }}\right| S\right\rangle_{1 \text { st term }} \\
& =-\frac{4 \pi \alpha}{Z} \sqrt{\frac{1}{3}} \int d^{3} x \psi_{P}^{*}(\boldsymbol{x}) \frac{1}{x^{2}} \psi_{S}(\boldsymbol{x}) \sum_{m} Y_{1}^{m}(\hat{x}) \boldsymbol{d}_{\mathcal{N}}^{m *} \hat{\mathcal{C}}_{0>}(x) \\
& =-\frac{4 \pi \alpha}{\sqrt{3} Z} \sum_{m} \int_{0}^{\infty} d x u_{P}(x)^{*} u_{S}(x) \int d \Omega_{x} Y_{1}^{\lambda *}(\hat{x}) Y_{1}^{m}(\hat{x}) Y_{0}(\hat{x}) \\
& \quad \times \boldsymbol{d}_{\mathcal{N}}^{m *} \int d^{3} y \theta(y-x) \hat{\rho}(\boldsymbol{y}) Y_{0}(\hat{y}) .
\end{aligned}
$$

The angular $d \Omega_{x}$ integral gives $\delta_{m \lambda} / \sqrt{4 \pi}$. To evaluate the radial integral we first write

$$
u_{P}(x)^{*} u_{S}(x)=\sum_{k \geqslant 1} c_{k} x^{k},
$$

where the $c_{k}$ are given by the appropriate products of the $a_{k}$ and $b_{k}$ (our procedure here is similar to that of Ref. [20]). Interchanging the order of integration and evaluating the 
integral over the electronic radial coordinate gives

$$
\begin{aligned}
& \left\langle P, \lambda\left|\hat{O}_{\text {Schiff }}^{\text {atomic }}\right| S\right\rangle_{1 \text { st term }} \\
& =-\frac{4 \pi \alpha}{\sqrt{3} Z} \frac{1}{4 \pi} \boldsymbol{d}_{\mathcal{N}}^{\lambda *} \int d^{3} y \hat{\rho}(\boldsymbol{y}) \sum_{k \geqslant 1} \frac{c_{k} y^{k+1}}{k+1} .
\end{aligned}
$$

Evaluating the second and third terms in the matrix element of $\hat{O}_{\text {Schiff }}^{\text {atom }}$ in a similar way leads to

$$
\begin{aligned}
\left\langle P, \lambda\left|\hat{O}_{\text {Schiff }}^{\text {atomic }}\right| S\right\rangle_{\text {terms } 1-3} & (4 \pi \alpha)\left(\frac{\sqrt{3}}{4 \pi}\right) \int d^{3} y \hat{\rho}(\boldsymbol{y}) \sum_{k \geqslant 1} \frac{c_{k} y^{k+1}}{(k+1)(k+4)}\left\{\boldsymbol{y}^{\lambda *}\right. \\
& \left.-\frac{(k+4)}{3 Z}\left(\boldsymbol{d}_{\mathcal{N}}^{\lambda *}-\frac{2(k+1) \sqrt{2 \pi}}{(k+4)}\left[\boldsymbol{d}_{\mathcal{N}} \otimes Y_{2}(\hat{y})\right]_{1}^{\lambda *}\right)\right\} .
\end{aligned}
$$

Note that in this expression $\boldsymbol{d}_{\mathcal{N}}^{\lambda}$ is the nuclear operator defined by Eq. (26).

The "local" nuclear Schiff moment operator $\hat{\boldsymbol{S}}_{L}^{\lambda}$ can be defined by requiring that

$$
-(4 \pi \alpha)\left\langle P, \lambda\left|\hat{\boldsymbol{S}}_{L} \cdot \nabla \delta^{(3)}(\boldsymbol{x})\right| S\right\rangle \equiv\left\langle P, \lambda\left|\hat{O}_{\text {Schiff }}^{\text {atomic }}\right| S\right\rangle_{\text {terms1-3 }} .
$$

Evaluating the left side of Eq. (76) yields

$$
-(4 \pi \alpha)\left\langle P, \lambda\left|\hat{\boldsymbol{S}}_{L} \cdot \nabla \delta^{(3)}(\boldsymbol{x})\right| S\right\rangle=(4 \pi \alpha)\left(\frac{\sqrt{3}}{4 \pi}\right) c_{1} \hat{\boldsymbol{S}}_{L}^{\lambda *} .
$$

Including for the moment only the first three terms in $\hat{O}_{\text {Schiff }}^{\text {atomic }}$ as in Eq. (75) gives

$$
\begin{aligned}
\hat{\boldsymbol{S}}_{L}^{\lambda}= & \int d^{3} y \hat{\rho}(\boldsymbol{y}) \sum_{k \geqslant 1} \frac{c_{k}}{c_{1}} \frac{y^{k+1}}{(k+1)(k+4)}\left\{\boldsymbol{y}^{\lambda}\right. \\
& \left.-\frac{(k+4)}{3 Z}\left(\boldsymbol{d}_{\mathcal{N}}^{\lambda}-\frac{2(k+1) \sqrt{2 \pi}}{(k+4)}\left[\boldsymbol{d}_{\mathcal{N}} \otimes Y_{2}(\hat{y})\right]_{1}^{\lambda}\right)\right\} .
\end{aligned}
$$

Retaining only the leading-order term (i.e., $k=1$ ) on the right-hand side of Eq. (78) yields our result for what is conventionally referred to as the Schiff moment operator, $\hat{S}^{\lambda}$ :

$$
\begin{aligned}
\hat{\boldsymbol{S}}^{\lambda}= & \frac{1}{10} \int d^{3} y \hat{\rho}(\boldsymbol{y}) y^{2}\left\{\boldsymbol{y}^{\lambda}\right. \\
& \left.-\frac{5}{3 Z}\left(\boldsymbol{d}_{\mathcal{N}}^{\lambda}-\frac{4 \sqrt{2 \pi}}{5}\left[\boldsymbol{d}_{\mathcal{N}} \otimes Y_{2}(\hat{y})\right]_{1}^{\lambda}\right)\right\} .
\end{aligned}
$$

Note that nuclear matrix elements of $\hat{S}_{L}^{\lambda}$ and $\hat{S}^{\lambda}$ will involve nuclear matrix elements of density-density correlations, since the last two terms on the right-hand side of Eqs. (78) and (79) contain the operator

$$
\boldsymbol{d}_{\mathcal{N}}^{\lambda}=\int d^{3} z \hat{\rho}(z) z^{\lambda}
$$

that multiplies the operators $\hat{\rho}(\boldsymbol{y}) y^{2}$ or $\hat{\rho}(\boldsymbol{y}) y^{2} Y_{2}^{M}(\hat{y})$.

The form of the operators in Eqs. (78) and (79) differs in two important respects from the operators previously used in the literature: (a) the presence of the final term involving the $Y_{2}(\hat{y})^{7}$ and (b) the present treatment of $\boldsymbol{d}_{\mathcal{N}}^{\lambda}$ as an operator rather than as a $c$-number. To illustrate the importance of this first difference, we consider the Schiff moment operator of the deuteron in the limit where only the nuclear polarization term (due to $\tilde{V}_{\text {int }}^{\text {nuc }}$ ) is retained. That is, we ignore the effects of PVTV one-body (e.g., the neutron and proton EDMs) and two-body charges and three-currents. Polarization terms (pol.) contribute via the one-body PCTC nuclear charge operator:

$$
\hat{\rho}(\boldsymbol{y})=\sum_{k} \delta^{(3)}\left(\boldsymbol{y}-\boldsymbol{r}_{k}\right) \tau_{p}^{k}, \quad \tau_{p}^{k}=\frac{1}{2}\left(1+\tau_{3}^{k}\right),
$$

where the sum is over both nucleons $(k=1,2)$ with coordinate $r_{k}$. Since only the proton contributes, we label the proton coordinate $r$ for simplicity. Substituting Eq. (81) into Eq. (79) leads to

$\left.\hat{\boldsymbol{S}}^{\lambda}\right|_{\text {deuteron }} ^{\text {pol. }}=\frac{1}{10} \tau_{p} r^{2}\left\{\left(1-\frac{5}{3}\right) \boldsymbol{r}^{\lambda}+\frac{4 \sqrt{2 \pi}}{3}\left[\boldsymbol{r} \otimes Y_{2}(\hat{r})\right]_{1}^{\lambda}\right\}$,

where we have performed the integrals over $\boldsymbol{y}$ and $z$. Using

$$
\left[\boldsymbol{r} \otimes Y_{2}(\hat{r})\right]_{1}^{\lambda}=-\frac{1}{\sqrt{2 \pi}} \boldsymbol{r}^{\lambda},
$$

we obtain

$$
\left.\hat{\boldsymbol{S}}^{\lambda}\right|_{\text {deuteron }} ^{\text {pol. }}=-\frac{1}{5} \tau_{p} r^{2} \boldsymbol{r}^{\lambda}
$$

where the coefficient $-1 / 5$ is a factor of three larger than had we omitted the $Y_{2}(\hat{y})$ term. Generalizing this argument to an arbitrary nucleus we obtain

$$
\begin{aligned}
\left.\hat{\boldsymbol{S}}^{\lambda}\right|^{\text {pol. }}= & \frac{1}{10} \sum_{k} \tau_{p}^{k} r_{k}^{2}\left\{\boldsymbol{r}_{k}^{\lambda}\right. \\
& \left.-\frac{5}{3 Z} \sum_{j} \tau_{p}^{j}\left(\boldsymbol{r}_{j}^{\lambda}-\frac{4 \sqrt{2 \pi}}{5}\left[\boldsymbol{r}_{j} \otimes Y_{2}\left(\hat{r}_{k}\right)\right]_{1}^{\lambda}\right)\right\} .
\end{aligned}
$$

Focusing on the terms in Eq. (85) for which $j=k$ and using $\tau_{p}^{k} \tau_{p}^{k}=\tau_{p}^{k}$ gives

$$
\left.\hat{\boldsymbol{S}}^{\lambda}\right|_{j=k} ^{\text {pol. }}=\frac{1}{10} \sum_{k} \tau_{p}^{k} r_{k}^{2} \boldsymbol{r}_{k}^{\lambda}\left\{1-\frac{5}{3 Z}\left(1+\frac{4}{5}\right)\right\},
$$

where the final $4 / 5$ arises from the $Y_{2}(\hat{y})$ term. For heavy nuclei, the effect of this correction will be suppressed by the factor of $1 / Z$, but its importance relative to the second term in Eq. (79) is of the same order. The ground-state expectation value of this odd-parity operator will be nonzero because of PVTV admixtures in the nuclear wave function.

The presence of the terms with $j \neq k$ points to the impact of treating $\boldsymbol{d}_{\mathcal{N}}$ as an operator rather than as a $c$-number. To see

\footnotetext{
${ }^{7}$ We note that a qudrupole term appears in Eq. (19) of Ref. [32]. However, this paper only deals with nucleon EDMs in the deuteron, and the quadruple term (and the monopole term too), unlike our approach, is still treated as a $c$-number.
} 
this, we write $\hat{\boldsymbol{S}}^{\lambda}$ as follows:

$$
\begin{aligned}
\hat{\boldsymbol{S}}^{\lambda}= & \frac{1}{10} \int d^{3} y \int d^{3} z \hat{\rho}(\boldsymbol{y}) y^{2}\left\{\boldsymbol{y}^{\lambda} \delta^{(3)}(\boldsymbol{z})-\frac{5}{3 Z} \hat{\rho}(\boldsymbol{z})\right. \\
& \left.\times\left(z^{\lambda}-\frac{4 \sqrt{2 \pi}}{5}\left[z \otimes Y_{2}(\hat{y})\right]_{1}^{\lambda}\right)\right\} .
\end{aligned}
$$

The product of charge density operators appearing in Eq. (87) implies that nuclear matrix elements of $\hat{\boldsymbol{S}}^{\lambda}$ involve a two-body correlation. A comparison of this result with one where $\boldsymbol{d}_{\mathcal{N}}$ is treated as a $c$-number can be made by inserting a complete set of intermediate nuclear states

$$
\mathcal{N}\langle\widetilde{\text { g.s. }}|\hat{\rho}(\boldsymbol{y}) \hat{\rho}(\boldsymbol{z})| \widetilde{\text { g.s. }}\rangle_{\mathcal{N}}=\sum_{n} \mathcal{N}\langle\widetilde{\text { g.s. }}|\hat{\rho}(\boldsymbol{y})| n\rangle\langle n|\hat{\rho}(\boldsymbol{z})| \widetilde{\text { g.s. }}\rangle_{\mathcal{N}}
$$

Treating $\boldsymbol{d}_{\mathcal{N}}$ as a $c$-number amounts to the assumption that this sum is effectively saturated by retaining only $|n\rangle=\mid$ g.s. $\rangle$. The numerical validity of this assumption is not at all clear, and certainly it should be explored in numerical calculations. This task goes beyond the scope of the present paper but will be explored in future work.

Additional contributions to the nuclear Schiff moment arise from S-P matrix elements of the last four terms in $\hat{O}_{\text {Schiff }}^{\text {atomic }}$ It is useful to distinguish contributions from these terms that are sensitive to electronic penetration inside the nucleus from those that are not. Following similar arguments to those used in deriving $\hat{\boldsymbol{S}}_{L}$ we obtain

$$
\begin{aligned}
& \left\langle P, \lambda\left|\hat{O}_{\text {Schiff }}^{\text {atomic }}\right| S\right\rangle_{\text {terms } 4-7} \\
& \equiv-(4 \pi \alpha)\langle P, \lambda| \boldsymbol{\Delta} \hat{\boldsymbol{S}}_{(1)} \cdot \delta^{(3)}(\boldsymbol{x}) \overleftrightarrow{\nabla}+\boldsymbol{\Delta} \hat{\boldsymbol{S}}_{(2)} \\
& \quad \dot{\odot}\left(\frac{1}{x^{3}} Y_{2}(\hat{x}) \overleftrightarrow{\nabla}\right)|S\rangle+\left\langle P, \lambda\left|\boldsymbol{\Delta} \hat{\boldsymbol{S}}_{(3)} \cdot \overleftrightarrow{\nabla}\right| S\right\rangle,
\end{aligned}
$$

where

$$
\begin{aligned}
\Delta \hat{\boldsymbol{S}}_{(1)}^{\lambda}= & -\frac{1}{3 Z} \sum_{j \geqslant 1, k \geqslant 0}\left(\frac{a_{k} b_{j}}{a_{0} b_{1}}\right) \int d^{3} z \int d^{3} y y^{j+k+1} \\
& \times[\hat{\rho}(\boldsymbol{z}), \hat{\rho}(\boldsymbol{y})]\left\{\frac{(j+2-k)}{(j+k+2)(j+k+1)} z^{\lambda}\right. \\
& \left.-\frac{2 \sqrt{2 \pi}(j-1-k)}{(j+k+4)(j+k-1)}\left[Y_{2}(\hat{y}) \otimes z\right]_{1}^{\lambda}\right\}, \\
\Delta \hat{\boldsymbol{S}}_{(2)}^{\lambda}= & -\frac{1}{5 Z}\left[\boldsymbol{d}_{\mathcal{N}}^{\lambda}, \hat{C}_{2}\right] \\
\boldsymbol{\Delta} \hat{\boldsymbol{S}}_{(3)}^{\lambda}= & \frac{1}{Z}\left[\boldsymbol{d}_{\mathcal{N}}^{\lambda}, H_{\mathrm{int}}^{\text {nuc }}\right] .
\end{aligned}
$$

Details pertaining to the derivation of $\Delta \hat{\boldsymbol{S}}_{(1)}^{\lambda}$ are given in Appendix B; as for $\Delta \hat{S}_{(2,3)}^{\lambda}$, they can be readily read by comparing Eqs. (65) and (89).

The operator $\Delta \hat{\boldsymbol{S}}_{(1)}^{\lambda}$ characterizes corrections to Schiff screening that arise when the nuclear EDM operator does not commute with the local (penetration), even- $J$ charge operator. The result is the presence of the commutator $[\hat{\rho}(z), \hat{\rho}(\boldsymbol{y})]$ in
Eq. (90). We expect that contributions from this commutator will be suppressed by powers of $v / c$, where $v$ is a typical nucleon velocity, since the leading-order (in $v / c$ ) parts of the one-body charge operator give a vanishing commutator. Inclusion of sub-leading one-body or two-body charge operators will not, in general, give a vanishing commutator since these sub-leading terms contain momentum and/or Pauli spin operators. Again, we defer a detailed analysis of the numerical importance of these effects to a future study and concentrate here on delineating the various contributions to the operator.

The operators $\Delta \hat{\boldsymbol{S}}_{(2,3)}^{\lambda}$ characterize long-distance screening corrections that occur when the nuclear EDM operator does not commute with either the quadrupole moment operator or the internal nuclear Hamiltonian. We expect the former to be suppressed by powers of $v / c$ for reasons similar to those for $\boldsymbol{\Delta} \hat{\boldsymbol{S}}_{(1)}^{\lambda}$. The commutator of $\boldsymbol{d}_{\mathcal{N}}^{\lambda}$ with $H_{\mathrm{int}}^{\text {nuc }}$ is, perhaps, more subtle. In general, the nuclear Hamiltonian contains both momentum- and spin-dependent forces. The commutator of the momentum and spin operators will not commute with $\boldsymbol{d}_{\mathcal{N}}^{\lambda}$, which generally contains both spin- and space-dependent components. As with $\Delta \hat{\boldsymbol{S}}_{(1)}^{\lambda}$ we defer a detailed analysis of these corrections to future study.

\section{A. Magnetic corrections}

Additional corrections to Schiff screening arise from the magnetic operators in $\hat{O}_{3,4,8,9}^{(\mathcal{N})}$. As with the Schiff operator terms, we collect the lowest-rank terms that can also induce electronic S-P transitions:

$$
\begin{aligned}
& {\left[\hat{O}_{3,4,8,9}^{(\mathcal{N})}\right]_{\text {lowest rank }}} \\
& =-\frac{4 \pi \alpha}{Z x^{3}}\left\{\left[Y_{2}(\hat{x}) \otimes \boldsymbol{\alpha}\right]_{1} \odot\left[\boldsymbol{d}_{\mathcal{N}} \otimes \sqrt{\frac{1}{6}}\left(\hat{M}_{1}-\hat{\mathcal{M}}_{1>}(x)\right)\right]_{1}\right. \\
& \quad-\left[Y_{2}(\hat{x}) \otimes \boldsymbol{\alpha}\right]_{2} \odot\left[\boldsymbol { d } _ { \mathcal { N } } \otimes \left(\sqrt{\frac{3}{10}}\left(\hat{M}_{1}+\hat{\mathcal{M}}_{1>}(x)\right)\right.\right. \\
& \left.\left.\left.+\sqrt{\frac{8}{15}} \hat{\mathcal{M}}_{1<}(x)\right)\right]\right]_{2} \\
& \quad-\left[Y_{0}(\hat{x}) \otimes \boldsymbol{\alpha}\right]_{1} \odot\left[\boldsymbol{d}_{\mathcal{N}} \otimes \sqrt{\frac{1}{3}} \hat{\mathcal{M}}_{1<}(x)\right]_{1} \\
& +\left[Y_{2}(\hat{x}) \otimes \boldsymbol{\alpha}\right]_{2} \odot \frac{1}{5}\left(\hat{M}_{2}+\hat{\mathcal{M}}_{2}(x)\right) \\
& \left.+\left(x\left[Y_{1}(\hat{x}) \otimes \boldsymbol{\alpha}\right]_{1} \stackrel{\leftrightarrow}{\nabla}\right) \odot\left[\boldsymbol{d}_{\mathcal{N}}, \frac{1}{3}\left(\hat{M}_{1}+\hat{\mathcal{M}}_{1}(x)\right)\right]\right\} \\
& \equiv \hat{O}_{\text {Schiff-mag }}^{\text {atomic }}
\end{aligned}
$$

The operator $\hat{O}_{\text {Schiff-mag }}^{\text {atomic }}$ contains two types of terms: (a) those arising from the "displacement" of the magnetic dipole interaction, containing the commutator of $\boldsymbol{d}_{\mathcal{N}}$ with the magnetic dipole operators, and (b) an explicit magnetic quadrupole interaction. Effects of the former type were identified in Schiff's original paper but have generally been neglected in 
subsequent work. For atoms with nuclear spins $I=1 / 2$, there is no magnetic quadrupole contribution.

The form of $\hat{O}_{\text {Schiff-mag }}^{\text {atomic }}$ simplifies when we consider the electronic matrix element, again under the factorization assumption of Eq. (1). To arrive at this simplification, we consider the symmetry properties of the relevant electronic operator

$$
\mathcal{O}_{j}^{m} \equiv\left[Y_{l}(\hat{x}) \otimes \alpha\right]_{j}^{m} .
$$

To proceed further, it is useful to consider the perturbed atomic states appearing in Eq. (51) and label them according to their angular momentum quantum numbers. We define the states $\left|\tilde{J}^{\prime} M_{J}=J\right\rangle_{e}$ entering Eq. (51) via

$$
\begin{aligned}
& \sum_{J^{\prime}}\left|\tilde{J}^{\prime} J\right\rangle_{e} \otimes|\mathrm{g} . \mathrm{s}\rangle_{\mathcal{N}} \\
& \quad \equiv \sum_{n} \frac{1}{E_{\mathrm{g} . \mathrm{s} .}-E_{n}} e^{\left\langle n\left|V_{\mathrm{ext}}^{(e)}\right| J J\right\rangle_{e}|n\rangle_{e} \otimes|\mathrm{g} . \mathrm{s}\rangle_{\mathcal{N}}}
\end{aligned}
$$

where $|J, J\rangle_{e}$ denotes the electronic ground state and $V_{\mathrm{ext}}^{(e)}$ contains the potential associated with the constant external electric field that mixes electronic states differing in orbital angular momentum by one unit. The electronic component of Eq. (51) involving a generic $\mathcal{O}_{j}^{m}$ operator then has the form

$$
\left[{ }_{e}\left\langle\tilde{J}^{\prime} J\left|\mathcal{O}_{j}^{0}\right| J J\right\rangle_{e}+{ }_{e}\left\langle J J\left|\mathcal{O}_{j}^{0}\right| \tilde{J}^{\prime} J\right\rangle_{e}\right]
$$

Now using the identity

$$
\langle a|\mathcal{O}| b\rangle=\left\langle T(b)\left|T \mathcal{O}^{\dagger} T^{-1}\right| T(a)\right\rangle,
$$

where $T$ is the anti-unitary time-reversal operator, along with the Hermiticity and time-reversal properties of $\mathcal{O}_{j}^{m}$ that

$$
\begin{aligned}
{\left[Y_{l} \otimes \boldsymbol{\alpha}\right]_{j}^{m \dagger} } & =(-1)^{l+1-j+m}\left[Y_{l} \otimes \boldsymbol{\alpha}\right]_{j}^{-m}, \\
T\left[Y_{l} \otimes \boldsymbol{\alpha}\right]_{j}^{m} T^{-1} & =(-1)^{l-j+m}\left[Y_{l} \otimes \boldsymbol{\alpha}\right]_{j}^{m},
\end{aligned}
$$

we can show that

$$
\begin{aligned}
& e_{e}\left\langle\tilde{J}^{\prime} J\left|\mathcal{O}_{j}^{0}\right| J J\right\rangle_{e} \\
& \quad=-{ }_{e}\left\langle T(J J)\left|\mathcal{O}_{j}^{0}\right| T\left(\tilde{J}^{\prime} J\right)\right\rangle_{e} \\
& \quad=-(-1)^{J+J+L}(-1)^{J^{\prime}+J+L^{\prime}}\left\langle J-J\left|\mathcal{O}_{j}^{0}\right| \tilde{J}^{\prime}-J\right\rangle \\
& \quad=-(-1)^{J^{\prime}-J+L^{\prime}+L}(-1)^{2 J+J^{\prime}+J+j}\left\langle J J\left|\mathcal{O}_{j}^{0}\right| \tilde{J}^{\prime} J\right\rangle,
\end{aligned}
$$

where the second line follows from the phase convention for the time-reversed state (using here the Condon-Shortley convention $^{8}$ ), and the third line follows from the symmetry properties of the $3-j$ symbol. Given the fact that $\left(L^{\prime}+L\right)$ is odd (parity change) and $2\left(J^{\prime}+J\right)$ is even, we have

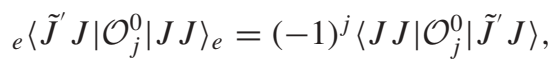

which means the sum, Eq. (96), can only be nonvanishing for $j=$ even. Thus, the $\left[Y_{0,2}(\hat{x}) \otimes \alpha\right]_{1}$ parts in Eq. (93) yield no

\footnotetext{
${ }^{8}$ Another popular phase convention is the one of Biedenharn-Rose, which introduces an extra " $i{ }^{L}$ " factor for $Y_{L}^{M}$. But the result is independent of phase convention.
}

contribution to the induced atomic dipole moment and we may work with the operator

$$
\begin{aligned}
\hat{O}_{\text {Schiff }- \text { mag }}^{\text {atomic }} & -\frac{4 \pi \alpha}{Z x^{3}}\left\{-\left[Y_{2}(\hat{x}) \otimes \boldsymbol{\alpha}\right]_{2} \odot\left[\boldsymbol { d } _ { \mathcal { N } } \otimes \left(\sqrt { \frac { 3 } { 1 0 } } \left(\hat{M}_{1}\right.\right.\right.\right. \\
= & \left.\left.\left.+\hat{\mathcal{M}}_{1>}(x)\right)+\sqrt{\frac{8}{15}} \hat{\mathcal{M}}_{1<}(x)\right)\right]_{2} \\
& +\left[Y_{2}(\hat{x}) \otimes \boldsymbol{\alpha}\right]_{2} \odot \frac{1}{5}\left[\hat{M}_{2}+\hat{\mathcal{M}}_{2}(x)\right] \\
& \left.+\left(x\left[Y_{1}(\hat{x}) \otimes \boldsymbol{\alpha}\right]_{1} \overleftrightarrow{\nabla}\right) \odot\left[\boldsymbol{d}_{\mathcal{N}}, \frac{1}{3}\left(\hat{M}_{1}+\hat{\mathcal{M}}_{1}(x)\right)\right]\right\} .
\end{aligned}
$$
term:

Several comments can be made regarding this $\hat{O}_{\text {Schiff-mag }}^{\text {atomic }}$

(i) As $\hat{O}_{\text {Schiff-mag }}^{\text {atomic }}$ involves interactions with static magnetic moments $M_{1}$ (through the combination with $\boldsymbol{d}_{\mathcal{N}}$ ) and $M_{2}$, they are not affected by the screening. Compared to the finite-size effects, which contain a suppression factor roughly of the order of $(\text { nuclear size })^{2} /(\text { atomic size })^{2} \sim \mathrm{fm}^{2} / a_{0}^{2} \sim 10^{-9}$, these magnetic effects are only suppressed by the typical hyperfine factor $\alpha^{2} m_{e} / m_{N} \sim 10^{-7}$. (Note that we have not taken any atomic or nuclear enhancement factor into account but have just used a pure dimensional analysis.) These could be potentially important, as already pointed out by Schiff [11] and others (see, e.g., $[17,19])$, particularly for the open-shell atoms.

(ii) The last term in $\hat{O}_{\text {Schiff-mag }}^{\text {atom }}$, unlike the rest, which are of quadrupole nature and need a nuclear spin equal or greater than 1 , contributes for any nucleus with spin. In fact, it has been identified in the original paper by Schiff [11] (who considered the hydrogen atom only) and later on studied by Hinds and Sandars [33] with a more refined expression. The latter authors find a non-negligible contribution from this term, about $20 \%$, to the EDM of the TIF molecule, assuming the proton EDM is the only CPV source. We emphasize that this term is a result of taking $\boldsymbol{d}_{\mathcal{N}}$ and the magnetic dipole $\hat{M}_{1}, \hat{\mathcal{M}}_{1}$ as operators: If one treats $d_{\mathcal{N}}$ as a $c$-number, this term does not arise.

\section{PRACTICAL CONSIDERATIONS}

In the foregoing analysis, we have laid out the structure of the atomic operators that describe the corrections to Schiff screening and that characterize the leading contributions to an atomic EDM arising from the relativistic motion of the electrons, the finite size and internal structure of the nucleus, and magnetic interactions between the atomic electrons and nucleus. In doing so, we have attempted to remain as general as possible without making specific reference to the atomic states. In this context, the leading contribution associated with finite nuclear size and internal structure is given by $\hat{O}_{\text {Schiff }}^{\text {atomic }}$ in Eq. (65). Given a complete basis of atomic states including the effects of P- and T-odd admixtures into the nuclear states of 
definite parity, one could in principle use $\hat{O}_{\text {Schiff }}^{\text {atomic }}$ to compute the energy shift of an atom in an external electric field via Eq. (51).

As a practical matter, it has been the convention to specify the computation of finite-size corrections by considering the effect of $\hat{O}_{\text {Schiff }}^{\text {atomic }}$ on mixing between electronic S and P states, making the factorization assumption of Eq. (1) and neglecting nuclear polarization corrections. To compare the implications of our formulation with previous analyses, we have derived an effective nuclear Schiff moment operator from $\hat{O}_{\text {Schiff }}^{\text {atomic }}$ under these assumptions and break down the matrix element as

$$
\begin{aligned}
& \left\langle P, \lambda\left|\hat{O}_{\text {Schiff }}^{\text {atomic }}\right| S\right\rangle \\
& =(-4 \pi \alpha)\langle P, \lambda|\left\{\hat{\boldsymbol{S}}_{L} \cdot \nabla \delta^{(3)}(\boldsymbol{x})+\Delta \hat{\boldsymbol{S}}_{(1)} \cdot \delta^{(3)}(\boldsymbol{x}) \overleftrightarrow{\nabla}\right. \\
& \left.\quad+\Delta \hat{\boldsymbol{S}}_{(2)} \odot \frac{1}{x^{3}} Y_{2}(\hat{x}) \overleftrightarrow{\nabla}\right\}|S\rangle+\left\langle P, \lambda\left|\Delta \hat{\boldsymbol{S}}_{(3)} \cdot \overleftrightarrow{\nabla}\right| S\right\rangle,
\end{aligned}
$$

where $\hat{\boldsymbol{S}}_{L}$ and $\boldsymbol{\Delta} \hat{\boldsymbol{S}}_{(1-3)}$ are given by Eqs. (78) and (90)-(92), respectively. We emphasize that different effective operators will apply for other atomic transitions. The derivation of the relevant operators starting from $\hat{O}_{\text {Schiff }}^{\text {atomic }}$ will follow logic similar to that in our derivation here for $\left\langle P, \lambda\left|\hat{O}_{\text {Schiff }}^{\text {atomic }}\right| S\right\rangle$. One can introduce a further level of approximation by specifying the sums in these equations to the leading terms, corresponding to retaining only the leading $x$ dependence of the electronic wave function near the origin. Quantifying the error introduced by making either the factorization approximation or retaining only the leading electronic $x$ dependence is an important task for future nuclear and atomic structure computations.

Having introduced these two approximations, we have obtained a form for $\left\langle P, \lambda\left|\hat{O}_{\text {Schiff }}^{\text {atomic }}\right| S\right\rangle$ that differs in several respects from the nuclear Schiff moment operator used previously in the literature. These differences arise primarily because we have reformulated Schiff's theorem entirely at the operator level, have retained the full nuclear operator dependence of the finite-size correction, and proceeded consistently within the framework of the spherical multipole expansion. In brief, the resulting differences with previous forms of the operator obtained in our approach are the following:

(i) the presence of the $Y_{2}(\hat{x})$ term and the product of nuclear density operators in Eqs. (78) and (79);

(ii) the presence of the commutator of nuclear density operators in Eqs. (90) and (91) that vanishes at leading order in $v / c$, but will not in general vanish at higher orders owing to the presence of spin and momentum dependences in the nuclear charge operators; and

(iii) the presence of the internal nuclear Hamiltonian in Eq. (92) that results from including the internal nuclear degrees of freedom as dynamical quantities in the atomic Hamiltonian.

We have illustrated the potential impact of including some of these new ingredients by studying the effect of the $Y_{2}(\hat{x})$ term in $\hat{S}$ in the deuteron, where it increases the magnitude of the Schiff moment by a factor of 3 at the one-body operator level, and in the diagonal part of the density-density operator matrix element for heavy nuclei, where its impact is $1 / Z$ suppressed. We have not, however, quantified the effect of nondiagonal terms and the corresponding nuclear excitations, those that arise at higher order in $v / c$, or those associated with the internal nuclear Hamiltonian. The result of future studies that quantify these contributions will determine the degree to which previous computations of the Schiff moment adequately characterize the leading nuclear correction to Schiff screening.

Additional corrections arise from the effect of magnetic interactions between the atomic electrons and nucleus. In principle, these corrections could be important for the theoretical interpretation of other atomic EDMs. To that end, we have worked out the leading form of the magnetic operator

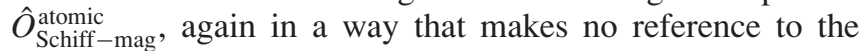
atomic states and later specifying to the situation of a simple direct product of nuclear and electronic states. Analyzing the quantitative impact of this operator will also be an interesting endeavor for future nuclear and atomic structure studies.

\section{ACKNOWLEDGMENTS}

We thank V. V. Flambaum and P. Vogel for useful discussions. Part of this work was supported by (i) the Dutch Stichting voor Fundamenteel Onderzoek der Materie (FOM) under program 48, TRI $\mu \mathrm{P}$ (CPL and RGET), (ii) the U.S. Department of Energy under Contract Nos. DE-AC52-06NA25396 (CPL), DE-FG02-05ER41361 (MJR-M), and DE-FG02-00ER-41132 (WCH), and (iii) the National Science Foundation under Grant No. PHY-0555674 (MJR-M).

\section{APPENDIX A \\ SYMMETRIZATION OF $[A(y) B(x), C(y) D(x)]$}

When evaluating Eq. (47) for the residual internal PVTV $e-\mathcal{N}$ interaction that evades the Schiff screening for $d_{\mathcal{N}}$, the commutator $\left[\boldsymbol{d}_{\mathcal{N}} \cdot \nabla, \Delta H_{0}\right]$ can be generically expressed as $[A(\boldsymbol{y}) B(\boldsymbol{x}), C(\boldsymbol{y}) D(\boldsymbol{x})]$ with $\mathcal{O}(\boldsymbol{y})$ and $\mathcal{O}(\boldsymbol{x})$ denoting operators acting upon nuclear and electronic Hilbert spaces, respectively. To obtain a manifestly Hermitian result, we employ the following identity:

$$
\begin{aligned}
& {[A(\boldsymbol{y})}B(\boldsymbol{x}), C(\boldsymbol{y}) D(\boldsymbol{x})] \\
&= \frac{1}{2}([B(\boldsymbol{x}), D(\boldsymbol{x})]\{A(\boldsymbol{y}), C(\boldsymbol{y})\}+\{B(\boldsymbol{x}), D(\boldsymbol{x})\} \\
&\quad \times[A(\boldsymbol{y}), C(\boldsymbol{y})]),
\end{aligned}
$$

where we have used the fact that operators depending on $\boldsymbol{x}$ and $\boldsymbol{y}$ commute: $[A(\boldsymbol{y}), B(\boldsymbol{x})]=0$, etc. As an illustrative example, we go through the terms in $\Delta H_{0}$ that involve static charge multipoles $C_{J}$ 's with $J \geqslant 2$. For this case, one takes

$$
\begin{aligned}
& A(\boldsymbol{y})=\boldsymbol{d}_{\mathcal{N}}, \quad B(\boldsymbol{x})=\nabla, \\
& C(\boldsymbol{y})=C_{J}^{M}, \quad D(\boldsymbol{x})=\frac{1}{x^{J+1}} Y_{J}^{M *}(\hat{x}),
\end{aligned}
$$

and uses the gradient formula

$$
\left[\nabla_{x}, \frac{1}{x^{J+1}} Y_{J}^{M *}(\hat{x})\right]=\sqrt{(J+1)(2 J+1)} \frac{1}{x^{J+2}} Y_{J J+11}^{M *}(\hat{x}),
$$


where $\boldsymbol{Y}_{J L 1}^{M}$ is the vector spherical harmonics. Then the commutator can be reduced as

$$
\begin{aligned}
& -\left[\boldsymbol{d}_{\mathcal{N}} \cdot \nabla_{x}, C_{J} \odot \frac{1}{x^{J+1}} Y_{J}(\hat{x})\right] \\
& =\sqrt{\frac{J+1}{2 J+3}} \frac{(2 J+1)}{x^{J+2}} Y_{J+1}(\hat{x}) \odot\left[\boldsymbol{d}_{\mathcal{N}} \otimes C_{J}\right]_{J+1}^{(\mathrm{sym})} \\
& \quad+\frac{1}{x^{J+1}} Y_{J}(\hat{x}) \stackrel{\leftrightarrow}{\odot} \dot{\ominus}\left[\boldsymbol{d}_{\mathcal{N}}, C_{J}\right]
\end{aligned}
$$

with the short-hand notations $[\ldots]^{\text {sym }}, \overleftrightarrow{\nabla}$, and $\odot$ being defined in Eqs. (62)-(64). This result leads to the $\hat{O}_{1}^{(\mathcal{N})}$ operator [i.e., Eq. (53)].

\section{APPENDIX B: DERIVATION OF $\Delta \hat{S}_{(1)}$}

In this appendix, we use the term involving $\mathcal{C}_{0}(x)$ in Eq. (65) (i.e., the fourth term) to illustrate the general procedure for obtaining $\Delta \hat{S}_{(1)}$. First, the electronic matrix element

$$
\begin{aligned}
\left\langle P, \lambda\left|\hat{O}_{\text {Schiff }}^{\text {atomic }}\right| S\right\rangle_{\text {term } 4}= & \langle P, \lambda| \frac{4 \pi \alpha}{Z x} Y_{0}(\hat{x}) \\
& \left.\otimes\left[\boldsymbol{d}_{\mathcal{N}}, \mathcal{C}_{0}(x)\right] \cdot \stackrel{\nabla}{\nabla}\right]|S\rangle
\end{aligned}
$$

can be easily evaluated with

$$
\begin{aligned}
& \int d^{3} x \frac{1}{x} Y_{0}(\hat{x}) \theta(y-x)\left(\frac{x}{y}-1\right) \frac{1}{2}\left(\psi_{P, \lambda}^{*} \nabla \psi_{S}-\psi_{S} \nabla \psi_{P, \lambda}\right) \\
& =\frac{\boldsymbol{e}_{\lambda}^{*}}{4 \sqrt{3 \pi}} \sum_{j \geqslant 1, k \geqslant 0} \frac{(j-k+2)}{(j+k+2)(j+k+1)} b_{j} a_{k} y^{j+k+1}, \quad \text { (B2) }
\end{aligned}
$$

so that

$$
\begin{aligned}
\left\langle P, \lambda\left|\hat{O}_{\text {Schiff }}^{\text {atomic }}\right| S\right\rangle_{\text {term } 4} & \frac{\alpha}{2 \sqrt{3} Z} \sum_{j \geqslant 1, k \geqslant 0} b_{j} a_{k} \int d^{3} z \int d^{3} y y^{j+k+1}[\hat{\rho}(z), \hat{\rho}(\boldsymbol{y})] \\
& \times \frac{(j-k+2)}{(j+k+2)(j+k+1)} z^{\lambda *} .
\end{aligned}
$$

Since this specific matrix element has been defined as [see Eq. (89)]

$$
\begin{aligned}
& \left\langle P, \lambda\left|\hat{O}_{\text {Schiff }}^{\text {atomic }}\right| S\right\rangle_{\text {terms } 4,6} \\
& \quad \equiv-(4 \pi \alpha)\left\langle P, \lambda\left|\Delta \hat{\boldsymbol{S}}_{(1)} \odot \delta^{(3)}(\boldsymbol{x}) \stackrel{\nabla}{\nabla}\right| S\right\rangle \\
& \quad=-(4 \pi \alpha)\left(\frac{\sqrt{3}}{4 \pi}\right) \frac{1}{2} b_{1} a_{0} \hat{\boldsymbol{S}}_{(1)}^{\lambda *},
\end{aligned}
$$

then the contribution of the $\mathcal{C}_{0}$ term to $\hat{\boldsymbol{S}}_{(1)}^{\lambda}$ becomes

$$
\begin{aligned}
\Delta \hat{\boldsymbol{S}}_{(1), \text { term } 4}^{\lambda} & -\frac{1}{3 Z} \sum_{j \geqslant 1, k \geqslant 0}\left(\frac{b_{j} a_{k}}{b_{1} a_{0}}\right) \int d^{3} z \int d^{3} y y^{j+k+1} \\
& \times[\hat{\rho}(\boldsymbol{z}), \hat{\rho}(\boldsymbol{y})] \frac{(j-k+2)}{(j+k+2)(j+k+1)} z^{\lambda} .
\end{aligned}
$$

Similar logic applies to the term involving $\mathcal{C}_{2}(x)$.
[1] C. A. Baker et al., Phys. Rev. Lett. 97, 131801 (2006).

[2] M. V. Romalis, W. C. Griffith, J. P. Jacobs, and E. N. Fortson, Phys. Rev. Lett. 86, 2505 (2001).

[3] A. D. Sakharov, Pisma Zh. Eksp. Teor. Fiz. 5, 32 (1967) [JETP Lett. 5, 24 (1967)].

[4] V. Cirigliano, S. Profumo, and M. J. Ramsey-Musolf, J. High Energy Phys. 07 (2006) 002.

[5] M. Pospelov and A. Ritz, Ann. Phys. (NY) 318, 119 (2005).

[6] R. J. Holt(private communication); see also J. R. Guest et al., Phys. Rev. Lett. 98, 093001 (2007) and http://www.int. washington.edu/talks/WorkShops/int_07_1/People/Guest_J/ Guest.pdf.

[7] K. P. Jungmann and L. Willmann (private communication); see also H. W. Wilschut and K. P. Jungmann, Nucl. Phys. News 17, 11 (2007).

[8] M. V. Romalis (private communication); see also S. K. Lee, http: //www.int.washington.edu/talks/WorkShops/int_07_1/People/ Lee_S.K/SeungKyunLee.pdf.

[9] T. E. Chupp (private communication); see also http://www. int.washington.edu/talks/WorkShops/int_07_1/People/Chupp_T/ Chupppdf.pdf.

[10] B. R. Heckel (private communication); see also W. C. Griffith, http://www.int.washington.edu/talks/WorkShops/int_07_1/ People/Griffith_W.C/griffith_HgEDM.pdf.

[11] L. I. Schiff, Phys. Rev. 132, 2194 (1963).

[12] E. M. Purcell and N. F. Ramsey, Phys. Rev. 78, 807 (1950).

[13] P. G. H. Sandars, J. Phys. B 1, 511 (1968).

[14] G. Feinberg, Trans. N. Y. Acad. Sci. 38, 26 (1977).

[15] O. P. Sushkov, V. V. Flambaum, and I. B. Khriplovich, Zh. Eksp. Teor. Fiz. 87, 1521 (1984) [Sov. Phys. JETP 60, 873 (1984)].
[16] E. Lindroth, B. W. Lynn, and P. G. H. Sandars, J. Phys. B 22, 559 (1989).

[17] I. B. Khriplovich and S. K. Lamoreaux, CP Violation Without Strangeness: Electric Dipole Moments of Particles, Atoms, and Molecules (Springer-Verlag, Berlin, 1997).

[18] J. Engel, J. L. Friar, and A. C. Hayes, Phys. Rev. C 61, 035502 (2000).

[19] P. G. H. Sandars, Contemp. Phys. 42, 97 (2001).

[20] V. V. Flambaum and J. S. M. Ginges, Phys. Rev. A 65, 032113 (2002).

[21] J. H. de Jesus and J. Engel, Phys. Rev. C 72, 045503 (2005).

[22] V. A. Dzuba, V. V. Flambaum, J. S. M. Ginges, and M. G. Kozlov, Phys. Rev. A 66, 012111 (2002).

[23] J. Bernabeu and C. Jarlskog, Nucl. Phys. B75, 59 (1974).

[24] J. Bernabeu and C. Jarlskog, Phys. Lett. B60, 197 (1976).

[25] J. L. Friar, Phys. Rev. C 16, 1540 (1977).

[26] W. C. Haxton, C.-P. Liu, and M. J. Ramsey-Musolf, Phys. Rev. Lett. 86, 5247 (2001).

[27] W. C. Haxton and C. E. Wieman, Annu. Rev. Nucl. Part. Sci. 51, 261 (2001).

[28] W. C. Haxton, E. M. Henley, and M. J. Musolf, Phys. Rev. Lett. 63, 949 (1989).

[29] P. Herczeg, Nucl. Phys. 75, 655 (1966).

[30] C. P. Liu and R. G. E. Timmermans, Phys. Rev. C 70, 055501 (2004).

[31] J. D. Jackson, Classical Electrodynamics (Wiley, New York, 1975).

[32] V. F. Dmitriev, I. B. Khriplovich, and R. A. Sen'kov, hepph/0504063.

[33] E. A. Hinds and P. G. H. Sandars, Phys. Rev. A 21, 471 (1980). 